Supporting Information for

\title{
Synthesis of Substituted Oxa- and Aza[3.2.1] and [4.3.1]bicyclics via an Unprecedented Molybdenum-Mediated 1,5-“Michael-Type” Reaction
}

\author{
By Yongqiang Zhang and Lanny S. Liebeskind*
}

Sanford S. Atwood Chemistry Center, Emory University, 1515 Dickey Drive, Atlanta, GA, 30322

\section{GENERAL METHODS}

Unless otherwise indicated, all ${ }^{1} \mathrm{H}$ and ${ }^{13} \mathrm{C}$ NMR spectra were recorded on a Varian Inova $400 \mathrm{MHz}\left(400 \mathrm{MHz}{ }^{1} \mathrm{H}, 100 \mathrm{MHz}{ }^{13} \mathrm{C}\right)$ or Varian Inova $600 \mathrm{MHz}\left(600 \mathrm{MHz}{ }^{1} \mathrm{H}, 150 \mathrm{MHz}{ }^{13} \mathrm{C}\right)$ at room temperature in $\mathrm{CDCl}_{3}$ with internal $\mathrm{CHCl}_{3}$ as the reference $\left(7.27 \mathrm{ppm}\right.$ for ${ }^{1} \mathrm{H}$ and $77.23 \mathrm{ppm}$ for ${ }^{13} \mathrm{C}$ ). IR spectra were recorded on ASI ReactIR ${ }^{\mathrm{R}} 1000$ FT-IR spectrometer, equipped with a silicon probe. Peaks are reported $\left(\mathrm{cm}^{-1}\right)$ with the following relative intensities: s (strong, 67-100\%), m (medium, 40-67\%), w (weak, 20-40\%) and br (broad). Melting points (mp) are uncorrected and were taken in open capillary tubes on a Thomas Hoover capillary melting point apparatus. Since almost all of the Tp molybdenum complexes decompose at about $180-200^{\circ} \mathrm{C}$ melting points are not significant and are not shown in the experimental section. Optical rotations were measured with Perkin-Elmer 241MC or Perkin-Elmer Model 341 polarimeters. Analytical thin-layer chromatography (TLC) was carried out on commercial Baker-flex plastic-supported silica gel plates (thickness: $200 \mu \mathrm{m}$ ) with fluorescent indicator (F-254). Visualization was accomplished by UV light or stained with 5\% phosphomolybdic acid (PMA) in ethanol. Flash column chromatography was performed with 32-63 $\mu \mathrm{m}$ silica gel. In some cases (as indicated) silica gel was first neutralized with $\mathrm{Et}_{3} \mathrm{~N}$ prior to chromatography. Dichloromethane (HPLC grade) and THF $(99 \%+)$ were purchased from EMD and Aldrich (respectively) and dried over $4 \AA$ molecular sieves before used. Dry diethyl ether was purchased from Mallinckrodt and used as received. Unless otherwise specified, all reactions were carried out under a nitrogen or argon atmosphere, and all reaction flasks were flamed or oven dried prior to use. The nomenclature for determining the chirality of the molybdenum complexes is straightward. ${ }^{1}$

\section{STARTING MATERIALS.}

All enones, $\mathrm{TiCl}_{4}\left(1.0 \mathrm{M}\right.$ in dichloromethane), and $\mathrm{NaOCH}_{3}$ (powder) were purchased from Aldrich; $t$-BuOK, and $\mathrm{KOSiMe}_{3}$ were purchased from Fluka. All chemicals were used as received. $( \pm)$-Dicarbonyl[hydridotris(1-pyrazolyl)borato][( $\left.\eta^{3}-2,3,4\right)-5$-oxo-5,6-dihydro-2H-pyran-2- 
yl]molybdenum $[( \pm)-1]^{2}$ (-)-dicarbonyl[hydridotris(1-pyrazolyl)borato][(2S)-( $\left.\eta^{3}-2,3,4\right)-5$-oxo-5,6dihydro-2H-pyran-2-yl]molybdenum $[(-)-1],^{2}(+)$-dicarbonyl[hydridotris(1-pyrazolyl)borato][(2R)$\left(\eta^{3}-2,3,4\right)-5$-oxo-5,6-dihydro-2H-pyran-2-yl]molybdenum $\quad[(+)-1],{ }^{2} \quad$ and $\quad( \pm)$ dicarbonyl[hydridotris(1-pyrazolyl)borato][( $\left.\eta^{3}-2,3,4\right)-5$-methoxy-1-benzoxycarbonyl-1,2dihydropyridin-2-yl]molybdenum ${ }^{3,4}$ were prepared according to literature procedures. Both (-)-1 and (+)-1 were obtained in $>99 \%$ ee by recrystallization from a mixture of $\mathrm{CH}_{2} \mathrm{Cl}_{2}$ and hexanes. The observed optical rotations were: [(-)-1, $-550^{\circ}$ (c 1.42, $\mathrm{CH}_{2} \mathrm{Cl}_{2}$ ); (-)-1, +555 (c 1.67, $\left.\left.\mathrm{CH}_{2} \mathrm{Cl}_{2}\right)\right]$. $( \pm)$-Dicarbonyl[hydridotris(1-pyrazolyl)borato][( $\left.\eta^{3}-2,3,4\right)$-1-benzoxycarbonyl-5-oxo-5,6-dihydro2H-pyridin-2-yl]molybdenum [( \pm -2] was synthesized in almost quantitative yield by the hydrolysis of a solution of ( \pm )-dicarbonyl[hydridotris(1-pyrazolyl)borato][( $\left.\eta^{3}-2,3,4\right)-5$-methoxy-1benzoxycarbonyl-1,2-dihydropyridin-2-yl]molybdenum ${ }^{3,4}$ in methanol with $0.75 \mathrm{~N} \mathrm{HCl}$ (aqueous). $( \pm)$-2: TLC $\left(\mathrm{R}_{f}=0.62\right.$, hexanes-EtOAc 1:1). IR $\left(\mathrm{cm}^{-1}\right): 3123(\mathrm{w}), 2957(\mathrm{w}), 2490(\mathrm{~m}), 1965$ (s), 1868 (s), 1706 (m), 1664 (m), 1505 (m), 1409 (s), 1305 (s), 1285 (s), 1220 (s), 1123 (s), 1054 (s). ${ }^{1} \mathrm{H}$ NMR (a mixture of two rotamers): $\delta 8.45(\mathrm{~d}, J=1.91 \mathrm{~Hz}, 0.4 \mathrm{H}), 8.42(\mathrm{~d}, J=1.91 \mathrm{~Hz}, 0.6 \mathrm{H})$, $8.31(\mathrm{~d}, J=1.91 \mathrm{~Hz}, 0.6 \mathrm{H}), 7.76(\mathrm{~d}, J=1.91 \mathrm{~Hz}, 0.4 \mathrm{H}), 7.74(\mathrm{~d}, J=1.91 \mathrm{~Hz}, 0.6 \mathrm{H}), 7.70(\mathrm{~d}, J=$ $1.91 \mathrm{~Hz}, 0.4 \mathrm{H}), 7.65(\mathrm{~d}, J=1.91 \mathrm{~Hz}, 0.6 \mathrm{H}), 7.62(\mathrm{~d}, J=1.91 \mathrm{~Hz}, 0.6 \mathrm{H}), 7.60(\mathrm{~d}, J=1.91 \mathrm{~Hz}$, $0.4 \mathrm{H}), 7.58(\mathrm{~d}, J=1.91 \mathrm{~Hz}, 0.4 \mathrm{H}), 7.47-7.52(\mathrm{~m}, 1.6 \mathrm{H}), 7.40-7.44(\mathrm{~m}, 2.0 \mathrm{H}), 7.27-7.38(\mathrm{~m}, 3.0 \mathrm{H})$, $7.22(\mathrm{dd}, J=6.35 \mathrm{~Hz}, J=1.90 \mathrm{~Hz}, 0.4 \mathrm{H}), 6.28-6.30(\mathrm{~m}, 1.6 \mathrm{H}), 6.22-6.24(\mathrm{~m}, 1.0 \mathrm{H}), 5.97(\mathrm{t}, J=$ $2.22 \mathrm{~Hz}, 0.4 \mathrm{H}), 5.27$ (AB quartet, $J=11.44 \mathrm{~Hz}, 0.4 \mathrm{H}), 5.24(\mathrm{~s}, 0.6 \mathrm{H}), 4.74-4.77(\mathrm{~m}, 1.0 \mathrm{H}), 4.09$ (t, $J=6.35 \mathrm{~Hz}, 0.6 \mathrm{H}), 3.98(\mathrm{t}, J=6.35 \mathrm{~Hz}, 0.4 \mathrm{H}), 3.41$ (AB quartet, $J=20.01 \mathrm{~Hz}, 0.4 \mathrm{H}), 3.39$ (AB quartet, $J=19.69 \mathrm{~Hz}, 0.6 \mathrm{H}) .{ }^{13} \mathrm{C}$ NMR: $\delta 225.2,224.7,222.8,222.1,193.7,193.0,154.8,154.0$, $147.5,147.4$, 144.7, 143.6, 141.6, 141.5, 136.7, 136.62, 136.48, 136.44, 135.7, 135.5, 135.0, 129.1, $128.9,128.8,128.7,128.4,128.0,106.36,106.34,106.1,106.0,94.0,92.4,69.1,68.3,64.7,64.4$, 64.1, 63.6, 48.1, 48.0.

\section{GENERAL PROCEDURE FOR MUKAIYAMA-MICHAEL ADDITIONS. ${ }^{5}$}

To a $\mathrm{CH}_{2} \mathrm{Cl}_{2}$ solution of the molybdenum complexes 1 and 2 (1.0 equiv) was successively added $\mathrm{Et}_{3} \mathrm{~N}$ (1.10-1.15 equiv) and $t-\mathrm{BuMe}_{2} \mathrm{SiOSO}_{2} \mathrm{CF}_{3}$ (TBSOTf, 1.10 equiv). The reaction mixture was stirred at room temperature for about $10 \mathrm{~min}$ and was then cooled to $-78{ }^{\circ} \mathrm{C}$. To this cold solution was rapidly added a premixed $\mathrm{CH}_{2} \mathrm{Cl}_{2}$ solution of the indicated enones (1.4 equiv) and titanium tetrachloride (1.3 equiv) via syringe. The indicated enones were first dissolved in $\mathrm{CH}_{2} \mathrm{Cl}_{2}$ and then cooled to $-78{ }^{\circ} \mathrm{C}$. To this cold solution was then added titanium tetrachloride $(1.0 \mathrm{M}$ 
solution in $\mathrm{CH}_{2} \mathrm{Cl}_{2}$ ) via syringe. The reaction mixture was stirred at the indicated temperature for the indicated time, quenched with $1 \mathrm{~mL}$ of water or saturated sodium bicarbonate aqueous solution at $-78{ }^{\circ} \mathrm{C}$, and then partitioned between brine and dichloromethane. The organic layer was separated, and the aqueous layer was extracted with dichloromethane. The organic layers were combined, dried with $\mathrm{Na}_{2} \mathrm{SO}_{4}$ and the solvent was removed under vacuum to afford crude products. The crude products were purified by column chromatography to give solid organic products. The ratio of isomers was determined by ${ }^{1} \mathrm{H}$ NMR of the crude product. (Note: the reaction yields depended significantly on the quality of titanium tetrachloride.)

\section{( \pm )-Dicarbonyl[hydridotris(1-pyrazolyl)borato][(2S,6S)-( $\left.\eta^{3}-2,3,4\right)-5-0 x 0-6-(3-0 x o b u t y l)-5,6-$}

dihydro-2H-pyran-2-yl]molybdenum, ( \pm )-3.

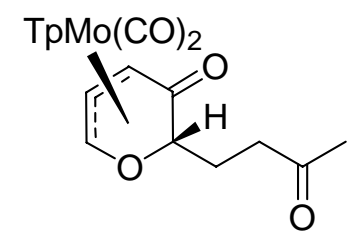

Following the general procedure, to a $10 \mathrm{~mL} \mathrm{CH}_{2} \mathrm{Cl}_{2}$ solution of the molybdenum complex 1 (0.92 g, $2.0 \mathrm{mmol})$ was successively added $\mathrm{Et}_{3} \mathrm{~N}(307 \mu \mathrm{L}, 2.2 \mathrm{mmol})$ and TBSOTf (506 $\mu \mathrm{L}, 2.2$ mmol). The reaction mixture was stirred at room temperature for about $10 \mathrm{~min}$ and was then cooled

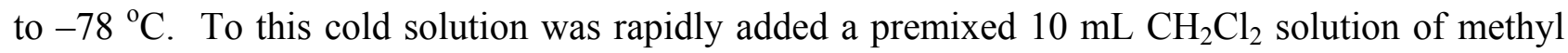
vinyl ketone MVK (196 mg, $2.8 \mathrm{mmol}$ ) and titanium tetrachloride (2.6 mL, $2.6 \mathrm{mmol})$ via syringe. The reaction mixture was stirred at $-78{ }^{\circ} \mathrm{C}$ for $5 \mathrm{~min}$ and quenched with $1 \mathrm{~mL}$ of saturated sodium bicarbonate aqueous solution at $-78{ }^{\circ} \mathrm{C}$. After work-up the crude product was purified by flash chromatography (1:1 hexanes-EtOAc) to give 3 as an orange solid $(1.00 \mathrm{~g}, 94 \%)$. $( \pm)-3$ : TLC $\left(\mathrm{R}_{f}=\right.$ 0.43, 1:1 hexanes-EtOAc). IR ( $\left.\mathrm{cm}^{-1}\right)$ : 3127 (w), 2934 (w), 2490 (m), 1957 (s), 1872 (s), 1714 (s), 1652 (s), 1505 (m), 1409 (s), 1305 (s), 1220 (s), 1050 (s). ${ }^{1} \mathrm{H}$ NMR: $\delta 8.51$ (d, J=2.23 Hz, 1H), $7.93(\mathrm{~d}, J=1.91 \mathrm{~Hz}, 1 \mathrm{H}), 7.65(\mathrm{~d}, J=2.23 \mathrm{~Hz}, 1 \mathrm{H}), 7.61(\mathrm{~d}, J=2.22 \mathrm{~Hz}, 1 \mathrm{H}), 7.53$ (d, $J=2.54 \mathrm{~Hz}$, $1 \mathrm{H}), 7.36(\mathrm{~d}, J=2.23 \mathrm{~Hz}, 1 \mathrm{H}), 7.34(\mathrm{dd}, J=4.76 \mathrm{~Hz}, J=2.22 \mathrm{~Hz}, 1 \mathrm{H}), 6.31(\mathrm{t}, J=2.22 \mathrm{~Hz}, 1 \mathrm{H})$, $6.27(\mathrm{t}, J=2.22 \mathrm{~Hz}, 1 \mathrm{H}), 6.22(\mathrm{t}, J=2.22 \mathrm{~Hz}, 1 \mathrm{H}), 4.77(\mathrm{dd}, J=6.03 \mathrm{~Hz}, J=2.22 \mathrm{~Hz}, 1 \mathrm{H}), 4.17$ $(\mathrm{dd}, J=6.04 \mathrm{~Hz}, J=4.76 \mathrm{~Hz}, 1 \mathrm{H}), 3.41(\mathrm{dd}, J=6.99 \mathrm{~Hz}, J=5.40 \mathrm{~Hz}, 1 \mathrm{H}), 2.54(\mathrm{~m}, 2 \mathrm{H}), 2.18(\mathrm{~s}$, 3H), 1.97-2.04 (m, 2H). ${ }^{13} \mathrm{C}$ NMR: $\delta 225.2,223.8,207.8,195.4,147.5,143.7,141.7,136.5,134.9$, 107.9, 106.4, 106.2, 105.9, 75.7, 68.6, 65.0, 39.0, 30.0, 26.8. HRMS (FAB) calcd for $\mathrm{C}_{20} \mathrm{H}_{22} \mathrm{BMoN}_{6} \mathrm{O}_{5}\left([\mathrm{M}+\mathrm{H}]^{+}\right): 535.0799$. Found: 535.0789. 
( \pm )-Dicarbonyl[hydridotris(1-pyrazolyl)borato][(2S,6S)-( $\left.\eta^{3}-2,3,4\right)-5-0 \times 0-6-(3-0 \times 0-1-$ phenylbutyl)-5,6-dihydro-2H-pyran-2-yl]molybdenum, ( \pm )-4.

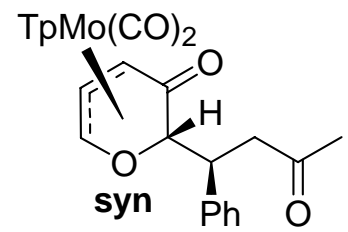

Following the general procedure, to a $5 \mathrm{~mL} \mathrm{CH}_{2} \mathrm{Cl}_{2}$ solution of the molybdenum complex 1 (500 mg, $1.09 \mathrm{mmol})$ was successively added $\mathrm{Et}_{3} \mathrm{~N}(174 \mu \mathrm{L}, 1.25 \mathrm{mmol})$ and TBSOTf $(275 \mu \mathrm{L}$, $1.20 \mathrm{mmol}$ ). The reaction mixture was stirred at room temperature for about $10 \mathrm{~min}$ and was then cooled to $-78{ }^{\circ} \mathrm{C}$. To this cold solution was rapidly added a premixed $5 \mathrm{~mL} \mathrm{CH}_{2} \mathrm{Cl}_{2}$ solution of trans-4-phenyl-3-buten-2-one $(204 \mathrm{mg}, 1.53 \mathrm{mmol})$ and titanium tetrachloride $(1.42 \mathrm{~mL}, 1.42$ mmol) via syringe. The reaction mixture was stirred at $-78{ }^{\circ} \mathrm{C}$ for $20 \mathrm{~min}$ and quenched with $1 \mathrm{~mL}$ of saturated sodium bicarbonate aqueous solution at $-78{ }^{\circ} \mathrm{C}$. After work-up the ${ }^{1} \mathrm{H}$ NMR spectrum of the crude product $(582 \mathrm{mg}$ ) showed a mixture of the two diastereomers (anti:syn =1:10) of 4 and the starting material 1 as well. Although 4 could not be separated from 1 the yield (71\%) was estimated by the ${ }^{1} \mathrm{H}$ NMR spectrum of the crude product. The pure syn isomer was obtained by recrystallization of the crude product from a mixture of dichloromethane and hexanes. Syn- $( \pm)-\mathbf{4}$ : TLC $\left(\mathrm{R}_{f}=\right.$ 0.54, 1:1 hexanes-EtOAc). IR $\left(\mathrm{cm}^{-1}\right): 3127(\mathrm{w}), 2490(\mathrm{~m}), 1961(\mathrm{~s}), 1872(\mathrm{~s}), 1718(\mathrm{~s})$, 1652 (s), 1505 (m), 1409 (s), 1305 (s), 1220 (s), 1123 (s), 1050 (s). ${ }^{1} \mathrm{H}$ NMR: $\delta 8.48$ (d, J= 2.22 $\mathrm{Hz}, 1 \mathrm{H}), 7.80(\mathrm{~d}, J=1.91 \mathrm{~Hz}, 1 \mathrm{H}), 7.58$ (d, $J=2.54 \mathrm{~Hz}, 2 \mathrm{H}), 7.54$ (d, $J=2.22 \mathrm{~Hz}, 1 \mathrm{H}), 7.49$ (d, $J=$ $2.22 \mathrm{~Hz}, 1 \mathrm{H}), 7.22-7.33(\mathrm{~m}, 6 \mathrm{H}), 7.37(\mathrm{dd}, J=4.63 \mathrm{~Hz}, J=1.91 \mathrm{~Hz}, 1 \mathrm{H}), 6.29(\mathrm{t}, J=2.22 \mathrm{~Hz}, 1 \mathrm{H})$, $6.22(\mathrm{t}, J=2.22 \mathrm{~Hz}, 1 \mathrm{H}), 6.15(\mathrm{t}, J=2.22 \mathrm{~Hz}, 1 \mathrm{H}), 4.42(\mathrm{dd}, J=6.03 \mathrm{~Hz}, J=2.23 \mathrm{~Hz}, 1 \mathrm{H}), 3.80$ $(\mathrm{td}, J=7.31 \mathrm{~Hz}, J=3.49 \mathrm{~Hz}, 1 \mathrm{H}), 3.64(\mathrm{dd}, J=6.35 \mathrm{~Hz}, J=4.76 \mathrm{~Hz}, 1 \mathrm{H}), 3.58(\mathrm{~d}, J=3.50 \mathrm{~Hz}$, $1 \mathrm{H}$ ), 2.98 (doublets of $\mathrm{AB}$ quartet, $\left.\mathrm{J}_{\mathrm{AB}}=17.25 \mathrm{~Hz}, J=7.31 \mathrm{~Hz}, 1 \mathrm{H}\right), 2.10(\mathrm{~s}, 3 \mathrm{H}) .{ }^{13} \mathrm{C} \mathrm{NMR}$ : $\delta 225.4,223.6,206.5,194.2,147.5,143.8,141.7,138.6,136.5,136.4,134.9,129.3,128.5,127.4$, 108.2, 106.5, 106.1, 105.8, 79.0, 69.2, 64.5, 45.7, 43.4, 30.5. HRMS (FAB) calcd for $\mathrm{C}_{25} \mathrm{H}_{26} \mathrm{BMoN}_{6} \mathrm{O}_{5}\left([\mathrm{M}+\mathrm{H}]^{+}\right): 611.1112$. Found: 611.1108 .

( \pm )-Dicarbonyl[hydridotris(1-pyrazolyl)borato][(2S,6S)-( $\left.\eta^{3}-2,3,4\right)-5-0 x 0-6-(3 '-0 x o c y c l o h e x y l)-$ 5,6-dihydro-2H-pyran-2-yl]molybdenum $\quad[( \pm)-5], \quad(+)$-dicarbonyl[hydridotris(1pyrazolyl)borato][(2R,6R,1'R)-( $\left.\eta^{3}-2,3,4\right)-5-0 x 0-6-\left(3^{\prime}-0 x o c y c l o h e x y l\right)-5,6-d i h y d r o-2 H$-pyran-2yl]molybdenum $[(+)-5]$ and (-)-dicarbonyl[hydridotris(1-pyrazolyl)borato][(2S,6S,1’ $S)-\left(\eta^{3}-\right.$ 2,3,4)-5-oxo-6-(3'-oxocyclohexyl)-5,6-dihydro-2H-pyran-2-yl]molybdenum [(-)-5]. 


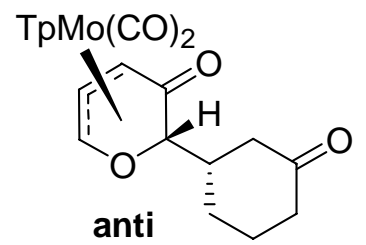

Following the general procedure, to a $12 \mathrm{~mL} \mathrm{CH}_{2} \mathrm{Cl}_{2}$ solution of the molybdenum complex 1 $(1.0 \mathrm{~g}, 2.17 \mathrm{mmol})$ was successively added $\mathrm{Et}_{3} \mathrm{~N}(353 \mu \mathrm{L}, 2.50 \mathrm{mmol})$ and TBSOTf (556 $\mu \mathrm{L}, 2.39$ mmol). The reaction mixture was stirred at room temperature for about $10 \mathrm{~min}$ and was then cooled to $-78{ }^{\circ} \mathrm{C}$. To this cold solution was rapidly added a premixed $12 \mathrm{~mL} \mathrm{CH}_{2} \mathrm{Cl}_{2}$ solution of cyclohexenone $(276 \mathrm{mg}, 3.04 \mathrm{mmol})$ and titanium tetrachloride $(2.82 \mathrm{~mL}, 2.82 \mathrm{mmol})$ via syringe. The reaction mixture was stirred at $-78{ }^{\circ} \mathrm{C}$ for $30 \mathrm{~min}$ and quenched with $1 \mathrm{~mL}$ of saturated sodium bicarbonate aqueous solution at $-78{ }^{\circ} \mathrm{C}$. After work-up the crude product was purified by flash chromatography (1:1 hexanes-EtOAc) to afford 5 as an orange solid (1.09 g, 91\%). The ${ }^{1} \mathrm{H}$ NMR spectrum of the crude product showed a mixture of the two diastereomers (anti:syn =40:1) of $( \pm$ )-5. Anti-( \pm )-5: TLC ( $\mathrm{R}_{f}=$ 0.59, 1:1 hexanes-EtOAc). IR ( $\left.\mathrm{cm}^{-1}\right): 3127(\mathrm{w}), 2945(\mathrm{w}), 2490(\mathrm{w}), 1961(\mathrm{~s})$, 1872 (s), 1710 (s), 1652 (s), 1505 (w), 1409 (s), 1305 (s), 1220 (s), 1123 (s), 1050 (s). ${ }^{1} \mathrm{H}$ NMR: $\delta$ $8.50(\mathrm{~d}, J=2.38 \mathrm{~Hz}, 1 \mathrm{H}), 7.90(\mathrm{~d}, J=1.91 \mathrm{~Hz}, 1 \mathrm{H}), 7.65(\mathrm{~d}, J=1.91 \mathrm{~Hz}, 1 \mathrm{H}), 7.63(\mathrm{~d}, J=2.38 \mathrm{~Hz}$, $1 \mathrm{H}), 7.60(\mathrm{~d}, J=2.38 \mathrm{~Hz}, 1 \mathrm{H}), 7.52(\mathrm{~d}, J=2.38 \mathrm{~Hz}, 1 \mathrm{H}), 7.39(\mathrm{dd}, J=4.28 \mathrm{~Hz}, J=1.91 \mathrm{~Hz}, 1 \mathrm{H})$, $6.30(\mathrm{t}, J=2.38 \mathrm{~Hz}, 1 \mathrm{H}), 6.26(\mathrm{t}, J=2.38 \mathrm{~Hz}, 1 \mathrm{H}), 6.22(\mathrm{t}, J=2.38 \mathrm{~Hz}, 1 \mathrm{H}), 4.79(\mathrm{dd}, J=6.19 \mathrm{~Hz}$, $J=1.91 \mathrm{~Hz}, 1 \mathrm{H}), 4.13(\mathrm{dd}, J=6.19 \mathrm{~Hz}, J=4.76 \mathrm{~Hz}, 1 \mathrm{H}), 3.37(\mathrm{~d}, J=2.38 \mathrm{~Hz}, 1 \mathrm{H}), 2.22-2.47(\mathrm{~m}$, 5H), 2.06-2.10 (m, 1H), 1.63-1.82 (m, 3H). ${ }^{13} \mathrm{C}$ NMR: $\delta 224.9,223.6,210.8,194.2,147.6,143.9$, 141.7, 136.61, 136.57, 135.0, 108.0, 106.6, 106.3, 106.0, 79.4, 70.0, 64.7, 41.54, 41.46, 41.2, 28.1, 24.7. HRMS (FAB) calcd for $\mathrm{C}_{22} \mathrm{H}_{23} \mathrm{BMoN}_{6} \mathrm{O}_{5}\left(\mathrm{M}^{+}\right)$: 560.0877. Found: 560.0905 .

Similar treatment of the chiral non-racemic (>99\% ee) molybdenum complex (2S)-(-)-1 (300 $\mathrm{mg}, 0.651 \mathrm{mmol})$ afforded $(-)-5$ as an orange solid $(288 \mathrm{mg}, 80 \%)$ in $99.3 \%$ ee $\left([\alpha]_{\mathrm{D}}=-479^{\circ}, c\right.$ 1.27, $\mathrm{CH}_{2} \mathrm{Cl}_{2}$ ).

Similar treatment of the chiral non-racemic ( $>99 \%$ ee) molybdenum complex $(2 R)-(+)-1(530$ $\mathrm{mg}, 1.17 \mathrm{mmol})$ afforded $(+)-5$ as an orange solid $(468 \mathrm{mg}, 71 \%)$ in $99.3 \%$ ee $\left([\alpha]_{\mathrm{D}}=+481^{\circ}, c\right.$ $\left.1.87, \mathrm{CH}_{2} \mathrm{Cl}_{2}\right)$.

( \pm )-Dicarbonyl[hydridotris(1-pyrazolyl)borato][(2S,6S)-( $\left.\eta^{3}-2,3,4\right)-5-0 x 0-6-(3-0 x 0 c y c l o p e n t y l)-$ 5,6-dihydro-2H-pyran-2-yl]molybdenum, ( \pm )-6. 


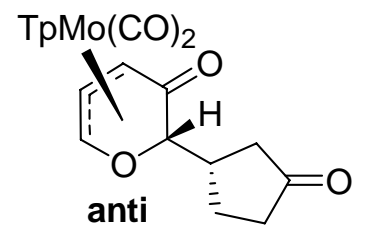

Following the general procedure, to a $5 \mathrm{~mL} \mathrm{CH}_{2} \mathrm{Cl}_{2}$ solution of the molybdenum complex 1 (500 mg, $1.09 \mathrm{mmol})$ was successively added $\mathrm{Et}_{3} \mathrm{~N}(174 \mu \mathrm{L}, 1.25 \mathrm{mmol})$ and TBSOTf $(275 \mu \mathrm{L}$, $1.20 \mathrm{mmol}$ ). The reaction mixture was stirred at room temperature for about $10 \mathrm{~min}$ and was then cooled to $-78{ }^{\circ} \mathrm{C}$. To this cold solution was rapidly added a premixed $5 \mathrm{~mL} \mathrm{CH}_{2} \mathrm{Cl}_{2}$ solution of cyclopentenone $(128 \mathrm{mg}, 1.53 \mathrm{mmol})$ and titanium tetrachloride $(1.42 \mathrm{~mL}, 1.42 \mathrm{mmol})$ via syringe. The reaction mixture was stirred at $-78{ }^{\circ} \mathrm{C}$ for $30 \mathrm{~min}$ and quenched with $1 \mathrm{~mL}$ of saturated sodium bicarbonate aqueous solution at $-78{ }^{\circ} \mathrm{C}$. After work-up the crude product was purified by flash chromatography (1:1 hexanes-EtOAc) to afford 6 as an orange solid (350 mg, 70\%). The ${ }^{1} \mathrm{H}$ NMR spectrum of the crude product showed a mixture of the two diastereomers (anti:syn $=7: 1$ ) of 6 .

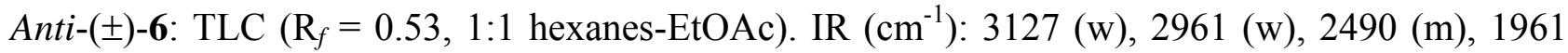
(s), 1872 (s), 1737 (s), 1652 (s), 1505 (m), 1409 (s), 1305 (s), 1220 (s), 1123 (s), 1050 (s). ${ }^{1} \mathrm{H}$ NMR: $\delta 8.52(\mathrm{~d}, J=1.91 \mathrm{~Hz}, 1 \mathrm{H}), 7.92(\mathrm{~d}, J=1.43 \mathrm{~Hz}, 1 \mathrm{H}), 7.65$ (d, $J=1.91 \mathrm{~Hz}, 1 \mathrm{H}), 7.63$ (d, $J=1.91$ $\mathrm{Hz}, 1 \mathrm{H}), 7.61(\mathrm{~d}, J=2.38 \mathrm{~Hz}, 1 \mathrm{H}), 7.53(\mathrm{~d}, J=2.38 \mathrm{~Hz}, 1 \mathrm{H}), 7.37$ (dd, $J=4.63 \mathrm{~Hz}, J=1.91 \mathrm{~Hz}$, $1 \mathrm{H}), 6.32(\mathrm{t}, J=2.38 \mathrm{~Hz}, 1 \mathrm{H}), 6.27(\mathrm{t}, J=1.91 \mathrm{~Hz}, 1 \mathrm{H}), 6.23(\mathrm{t}, J=2.38 \mathrm{~Hz}, 1 \mathrm{H}), 4.80(\mathrm{dd}, J=$ $6.19 \mathrm{~Hz}, J=1.91 \mathrm{~Hz}, 1 \mathrm{H}), 4.15(\mathrm{dd}, J=6.19 \mathrm{~Hz}, J=4.76 \mathrm{~Hz}, 1 \mathrm{H}), 3.48(\mathrm{~d}, J=2.86 \mathrm{~Hz}, 1 \mathrm{H}), 2.81-$ $2.84(\mathrm{~m}, 1 \mathrm{H}), 2.08-2.41(\mathrm{~m}, 5 \mathrm{H}), 1.93-1.98(\mathrm{~m}, 1 \mathrm{H}) .{ }^{13} \mathrm{C}$ NMR: $\delta 224.8,223.6,218.5,194.3,147.6$, $143.9,141.7,136.6,135.0,107.7,106.6,106.3,106.1,78.1,69.9,64.7,39.3,39.0,38.2,25.9$. HRMS (FAB) calcd for $\mathrm{C}_{21} \mathrm{H}_{21} \mathrm{BMoN}_{6} \mathrm{O}_{5}\left(\mathrm{M}^{+}\right)$: 546.0721. Found: 546.0734.

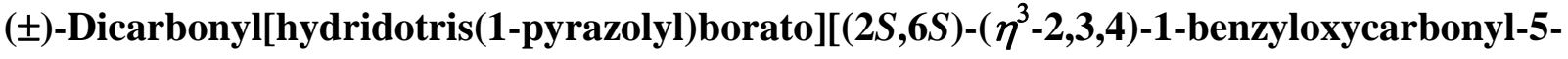
oxo-6-(3-oxobutyl)-5,6-dihydro-2H-pyridin-2-yl]molybdenum, ( \pm )-7.

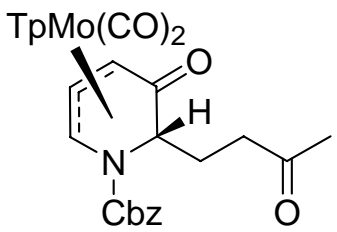

Following the general procedure, to a $10 \mathrm{~mL} \mathrm{CH}_{2} \mathrm{Cl}_{2}$ solution of the molybdenum complex 2 $(0.90 \mathrm{~g}, 1.51 \mathrm{mmol})$ was successively added $\mathrm{Et}_{3} \mathrm{~N}(231 \mu \mathrm{L}, 1.66 \mathrm{mmol})$ and TBSOTf $(382 \mu \mathrm{L}, 1.66$ $\mathrm{mmol})$. The reaction mixture was stirred at room temperature for about $10 \mathrm{~min}$ and was then cooled

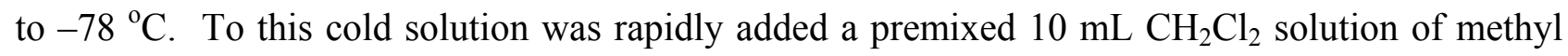
vinyl ketone MVK (151 mg, $2.11 \mathrm{mmol})$ and titanium tetrachloride (1.97 mL, $1.97 \mathrm{mmol})$ via syringe. The reaction mixture was stirred at $-78{ }^{\circ} \mathrm{C}$ for $10 \mathrm{~min}$ and quenched with $1 \mathrm{~mL}$ of 
saturated sodium bicarbonate aqueous solution at $-78{ }^{\circ} \mathrm{C}$. After work-up the crude product was purified by flash chromatography (1:1 hexanes-EtOAc) to afford 7 as an orange solid (908 $\mathrm{mg}$, 90.3\%). ( \pm )-7: TLC $\left(\mathrm{R}_{f}=0.58,1: 1\right.$ hexanes-EtOAc). IR ( $\left.\mathrm{cm}^{-1}\right): 3123(\mathrm{w}), 2957(\mathrm{w}), 2490(\mathrm{~m}), 1969$ (s), 1864 (s), 1706 (m), 1664 (m), 1505 (m), 1409 (s), 1305 (s), 1220 (s), 1123 (s), 1050 (s). ${ }^{1} \mathrm{H}$ NMR (a mixture of two rotamers): $\delta 8.56(\mathrm{~d}, J=1.91 \mathrm{~Hz}, 0.5 \mathrm{H}), 8.42(\mathrm{~d}, J=1.91 \mathrm{~Hz}, 0.5 \mathrm{H}), 8.37$ $(\mathrm{d}, J=1.91 \mathrm{~Hz}, 0.5 \mathrm{H}), 7.70(\mathrm{~d}, J=1.91 \mathrm{~Hz}, 0.5 \mathrm{H}), 7.67(\mathrm{~s}, 1 \mathrm{H}), 7.64(\mathrm{~d}, J=2.22 \mathrm{~Hz}, 0.5 \mathrm{H}), 7.62$ $(\mathrm{d}, J=2.23 \mathrm{~Hz}, 0.5 \mathrm{H}), 7.58$ (d, $J=2.44 \mathrm{~Hz}, 0.5 \mathrm{H}), 7.53$ (d, $J=2.22 \mathrm{~Hz}, 0.5 \mathrm{H}), 7.48-7.52(\mathrm{~m}, 2 \mathrm{H})$, 7.40-7.43 (m, 1H), 7.35-7.38 (m, 2H), $7.33(\mathrm{dd}, J=6.35 \mathrm{~Hz}, J=1.50 \mathrm{~Hz}, 0.5 \mathrm{H}), 7.08(\mathrm{dd}, J=6.35$ $\mathrm{Hz}, J=1.59 \mathrm{~Hz}, 0.5 \mathrm{H}), 6.27-6.30(\mathrm{~m}, 1.5 \mathrm{H}), 6.23(\mathrm{t}, J=2.22 \mathrm{~Hz}, 0.5 \mathrm{H}), 6.21(\mathrm{t}, J=2.22 \mathrm{~Hz}, 0.5 \mathrm{H})$, $5.81(\mathrm{t}, J=2.22 \mathrm{~Hz}, 0.5 \mathrm{H}), 5.24(\mathrm{~s}, 0.5 \mathrm{H}), 5.23$ (AB quartet, $J=11.43 \mathrm{~Hz}, 0.5 \mathrm{H}), 4.74$ (dd, $J=6.03$ $\mathrm{Hz}, J=1.90 \mathrm{~Hz}, 0.5 \mathrm{H}), 4.71(\mathrm{dd}, J=6.04 \mathrm{~Hz}, J=1.91 \mathrm{~Hz}, 0.5 \mathrm{H}), 4.03(\mathrm{t}, J=6.19 \mathrm{~Hz}, 0.5 \mathrm{H}), 3.91$ $(\mathrm{t}, J=6.19 \mathrm{~Hz}, 0.5 \mathrm{H}), 3.69(\mathrm{t}, J=6.67 \mathrm{~Hz}, 0.5 \mathrm{H}), 3.61(\mathrm{dd}, J=6.99, J=5.08 \mathrm{~Hz}, 0.5 \mathrm{H}), 2.44-2.71$ $(\mathrm{m}, 2 \mathrm{H}), 2.17(\mathrm{~s}, 1.5 \mathrm{H}), 2.10(\mathrm{~s}, 1.5 \mathrm{H}), 2.05-2.15(\mathrm{~m}, 2 \mathrm{H}) .{ }^{13} \mathrm{C}$ NMR: $\delta 225.9,225.2,222.9,221.7$, 207.8, 207.4, 197.5, 196.9, 154.9, 153.9, 147.6, 147.5, 147.1, 144.6, 140.8, 140.5, 136.7, 136.6, $136.46,136.39,135.7,135.4,135.09,135.04,129.00,128.96,128.8,128.6,128.4,106.5,106.4$, 106.05, 106.01, 98.4, 95.3, 69.4, 68.7, 65.2, 64.5, 59.6, 58.8, 57.5, 57.4, 39.6, 39.1, 30.1, 27.3, 26.7. HRMS (FAB) calcd for $\mathrm{C}_{28} \mathrm{H}_{28} \mathrm{BMoN}_{7} \mathrm{O}_{6}\left(\mathrm{M}^{+}\right)$: 667.1248, found: 667.1245.

\section{GENERAL PROCEDURE FOR METAL-MEDIATED 1,5-MICHAEL-TYPE ADDITIONS.}

To a $\mathrm{CH}_{2} \mathrm{Cl}_{2}$ solution of the molybdenum complex 3-7 (1.0 equiv) was added the indicated amount of base (3.0-6.0 equiv). The reaction mixture was stirred at room temperature until TLC monitoring of the reaction mixture indicated the disappearance of the starting material. The indicated amount of Meerwein salt $\mathrm{Me}_{3} \mathrm{OBF}_{4}$ (2.5-5.0 equiv) was added as a solid, and the mixture was then stirred at room temperature for the indicated time. The reaction mixture was directly poured onto a short pad of silica gel. Elution with 50\% ethyl acetate in hexanes, concentration and careful chromatographic purification afforded products 8-15 as yellow solids. Unless the isomers could be separated by flash column chromatography, the ratio of isomers was determined by ${ }^{1} \mathrm{H}$ NMR of the crude product.

( \pm )-Dicarbonyl[hydridotris(1-pyrazolyl)borato][(1S,2S,5R,6R)-( $\left.\eta^{3}-2,3,4\right)$-6-acetyl-2-methoxy8-oxabicyclo[3.2.1]oct-3-en-2-yl]molybdenum [( \pm )-8-exo] and ( \pm -dicarbonyl[hydridotris(1pyrazolyl)borato][(1S,2S,5R,6S)-( $\eta^{3}$-2,3,4)-6-acetyl-2-methoxy-8-oxabicyclo[3.2.1]oct-3-en-2yl]molybdenum [( \pm )-8-endo]. 

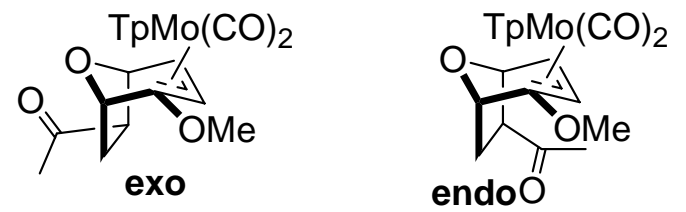

Following the general procedure, to a solution of the molybdenum complex $3(210 \mathrm{mg}$, $0.395 \mathrm{mmol})$ in $\mathrm{CH}_{2} \mathrm{Cl}_{2}(20 \mathrm{~mL})$ was added solid sodium methoxide (64 $\left.\mathrm{mg}, 1.18 \mathrm{mmol}\right)$. The reaction mixture was stirred at room temperature for $5 \mathrm{~h} . \mathrm{Me}_{3} \mathrm{OBF}_{4}(172 \mathrm{mg}, 1.18 \mathrm{mmol})$ was added as a solid and the mixture was then stirred at room temperature for $4 \mathrm{~h}$. Purification by flash chromatography afforded the molybdenum complexes $( \pm)$-8-exo $(151 \mathrm{mg}, 74 \%)$ and $( \pm)$-8-endo (20 mg, 9\%). ( \pm )-8-exo: TLC $\left(\mathrm{R}_{f}=0.57,1: 1\right.$ hexanes-EtOAc). IR $\left(\mathrm{cm}^{-1}\right): 3127(\mathrm{w}), 2922(\mathrm{~m}), 2853(\mathrm{w})$, 2482 (m), 1926 (s), 1837 (s), 1710 (s), 1505 (m), 1409 (s), 1305 (s), 1212 (s), 1050(s). ${ }^{1}$ H NMR: $\delta$ $8.46(\mathrm{~d}, J=1.91 \mathrm{~Hz}, 1 \mathrm{H}), 7.95(\mathrm{~d}, J=1.43 \mathrm{~Hz}, 1 \mathrm{H}), 7.62$ (t, $J=2.28 \mathrm{~Hz}, 2 \mathrm{H}), 7.59$ (d, $J=1.43 \mathrm{~Hz}$, $1 \mathrm{H}), 7.51(\mathrm{~d}, J=2.38 \mathrm{~Hz}, 1 \mathrm{H}), 6.24(\mathrm{t}, J=1.91 \mathrm{~Hz}, 1 \mathrm{H}), 6.22(\mathrm{t}, J=1.91 \mathrm{~Hz}, 1 \mathrm{H}), 6.16(\mathrm{t}, J=1.91$ $\mathrm{Hz}, 1 \mathrm{H}), 4.45(\mathrm{~d}, J=5.71 \mathrm{~Hz}, 1 \mathrm{H}), 4.38(\mathrm{~d}, J=2.38 \mathrm{~Hz}, 1 \mathrm{H}), 3.83(\mathrm{dd}, J=7.62 \mathrm{~Hz}, J=2.86 \mathrm{~Hz}$, $1 \mathrm{H}), 3.49$ (d, $J=7.62 \mathrm{~Hz}, 1 \mathrm{H}), 3.37$ (dd, $J=8.57 \mathrm{~Hz}, J=4.76 \mathrm{~Hz}, 1 \mathrm{H}), 3.24$ (s, 3H), 2.43 (dt, $J=$ $12.38 \mathrm{~Hz}, J=5.24 \mathrm{~Hz}, 1 \mathrm{H}), 2.29(\mathrm{dd}, J=12.39 \mathrm{~Hz}, J=9.05 \mathrm{~Hz}, 1 \mathrm{H}), 2.22(\mathrm{~s}, 3 \mathrm{H}) .{ }^{13} \mathrm{C} \mathrm{NMR}$ : $\delta 229.2,227.8,207.6,146.4,144.8,140.3,136.5,136.1,134.5,133.3,105.8,105.61,105.57,76.9$, 75.7, 60.6, 58.9, 56.4, 52.7, 35.1, 27.7. HRMS (FAB) calcd for $\mathrm{C}_{21} \mathrm{H}_{23} \mathrm{BMoN}_{6} \mathrm{O}_{5}\left(\mathrm{M}^{+}\right): 548.0877$. Found: 548.0853. ( \pm )-8-endo: TLC $\left(\mathrm{R}_{f}=0.69\right.$, hexanes-EtOAc 1:1). IR ( $\left.\mathrm{cm}^{-1}\right): 3127(\mathrm{w}), 2976(\mathrm{w})$, 2945(w), 2482 (w), 1926 (s), 1841 (s), 1710 (s), 1505 (m), 1409 (s), 1305 (s), 1216 (s), 1050 (s). ${ }^{1} \mathrm{H}$ NMR: $\delta 8.46(\mathrm{~d}, J=1.90 \mathrm{~Hz}, 1 \mathrm{H}), 7.94(\mathrm{~d}, J=1.91 \mathrm{~Hz}, 1 \mathrm{H}), 7.60(\mathrm{t}, J=1.91 \mathrm{~Hz}, 2 \mathrm{H}), 7.50(\mathrm{~d}, J=$ $1.91 \mathrm{~Hz}, 1 \mathrm{H}), 7.42(\mathrm{~d}, J=1.91 \mathrm{~Hz}, 1 \mathrm{H}), 6.25(\mathrm{t}, J=2.23 \mathrm{~Hz}, 1 \mathrm{H}), 6.18(\mathrm{t}, J=2.23 \mathrm{~Hz}, 1 \mathrm{H}), 6.15(\mathrm{t}$, $J=2.23 \mathrm{~Hz}, 1 \mathrm{H}), 4.62(\mathrm{dd}, J=6.35 \mathrm{~Hz}, J=1.90 \mathrm{~Hz}, 1 \mathrm{H}), 4.31(\mathrm{~d}, J=6.03 \mathrm{~Hz}, 1 \mathrm{H}), 3.59(\mathrm{ddd}, J=$ $10.35 \mathrm{~Hz}, J=6.67 \mathrm{~Hz}, J=4.45 \mathrm{~Hz}, 1 \mathrm{H}$ ), 3.46 (AB quartet, $J=7.67 \mathrm{~Hz}$, doublets of the AB left part, $J=4.76 \mathrm{~Hz}, 2 \mathrm{H}), 3.24(\mathrm{~s}, 3 \mathrm{H}), 2.63(\mathrm{dd}, J=12.71 \mathrm{~Hz}, J=4.45 \mathrm{~Hz}, 1 \mathrm{H}), 2.40$ (s, 3H), 2.14 (ddd, $J=12.38 \mathrm{~Hz}, J=10.48 \mathrm{~Hz}, J=6.36 \mathrm{~Hz}, 1 \mathrm{H}) .{ }^{13} \mathrm{C}$ NMR: $\delta 230.0,228.0,205.6,146.4,144.8$, $140.1,136.5,136.1,134.5,133.8,105.8,105.5,75.8,75.7,58.0,56.6,55.6,53.9,32.7,31.2$. HRMS (FAB) calcd for $\mathrm{C}_{21} \mathrm{H}_{23} \mathrm{BMoN}_{6} \mathrm{O}_{5}\left(\mathrm{M}^{+}\right)$: 548.0877. Found: 548.0887.

Following the general procedure, to a solution of the molybdenum complex $3(154 \mathrm{mg}$, $0.289 \mathrm{mmol})$ in $\mathrm{CH}_{2} \mathrm{Cl}_{2}(20 \mathrm{~mL})$ was added solid potassium trimethylsilanolate $(111 \mathrm{mg}, 0.87$ mmol). The reaction mixture was stirred at room temperature for $1 \mathrm{~h} . \mathrm{Me}_{3} \mathrm{OBF}_{4}(107 \mathrm{mg}, 0.723$ mmol) was added as a solid and the mixture was then stirred at room temperature for $40 \mathrm{~min}$. Purification by flash chromatography afforded the molybdenum complexes $( \pm)$-8-exo (150 mg, 95\%) and ( \pm )-8-endo (5 mg, 3\%). 
(土)-Dicarbonyl[hydridotris(1-pyrazolyl)borato][(1R,6S,7S)-( $\left.\eta^{3}-7,8,9\right)-7-$ methoxy-3-oxo-10oxabicyclo[4.3.1]dec-8-en-7-yl]molybdenum [( \pm -13].

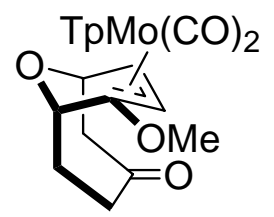

Following the general procedure, to a solution of the molybdenum complex 3 (266 mg, 0.5 $\mathrm{mmol})$ in $\mathrm{CH}_{2} \mathrm{Cl}_{2}(13 \mathrm{~mL})$ was added solid potassium $t$-butoxide (140 $\left.\mathrm{mg}, 1.25 \mathrm{mmol}\right)$. The reaction mixture was stirred at room temperature for $1 \mathrm{~h} . \mathrm{Me}_{3} \mathrm{OBF}_{4}(185 \mathrm{mg}, 1.25 \mathrm{mmol})$ was added as a solid and the mixture was then stirred at room temperature for $1 \mathrm{~h}$. Purification by flash chromatography afforded the molybdenum complex $( \pm)$-8-endo $(6 \mathrm{mg}, 2.2 \%)$ and an inseparable isomeric mixture of $( \pm)$-8-exo and $( \pm)-\mathbf{1 3}(198 \mathrm{mg}, 73 \%)$. The ${ }^{1} \mathrm{H}$ NMR spectrum of the inseparable mixture showed a 2:3 ratio of 8-exo:13. The pure isomer $\mathbf{1 3}$ was obtained by recrystallization from dichloromethane and hexanes. $( \pm)-13$ : TLC $\left(\mathrm{R}_{f}=0.57,1: 1\right.$ hexanes-EtOAc). IR (cm $\left.{ }^{-1}\right): 3127$ (w), 2941(w), 2482 (w), 1926 (s), 1837 (s), 1698 (s), 1505 (m), 1409 (m), 1305 (m), 1229 (s), 1050 (s). ${ }^{1} \mathrm{H}$ NMR: $\delta 8.44$ (d, $\left.J=2.38 \mathrm{~Hz}, 1 \mathrm{H}\right), 7.83$ (d, $\left.J=1.91 \mathrm{~Hz}, 1 \mathrm{H}\right), 7.63$ (d, $J$ $=2.39 \mathrm{~Hz}, 1 \mathrm{H}), 7.62(\mathrm{~d}, J=1.90 \mathrm{~Hz}, 1 \mathrm{H}), 7.56(\mathrm{~d}, J=1.91 \mathrm{~Hz}, 1 \mathrm{H}), 7.50(\mathrm{~d}, J=2.38 \mathrm{~Hz}, 1 \mathrm{H}), 6.24$ (t, $J=2.38 \mathrm{~Hz}, 1 \mathrm{H}), 6.22(\mathrm{t}, J=1.90 \mathrm{~Hz}, 1 \mathrm{H}), 6.17(\mathrm{t}, J=1.90 \mathrm{~Hz}, 1 \mathrm{H}), 5.39(\mathrm{dd}, J=5.24 \mathrm{~Hz}, J=$ $2.36 \mathrm{~Hz}, 1 \mathrm{H}), 4.07$ (t, $J=3.81 \mathrm{~Hz}, 1 \mathrm{H}), 3.77(\mathrm{~s}, 2 \mathrm{H}), 3.32(\mathrm{dd}, J=13.34 \mathrm{~Hz}, J=3.81 \mathrm{~Hz}, 1 \mathrm{H}), 2.98$ (s, 3H), $2.90(\mathrm{dd}, J=13.34 \mathrm{~Hz}, J=4.28 \mathrm{~Hz}, 1 \mathrm{H}), 2.55-2.59(\mathrm{~m}, 2 \mathrm{H}), 2.39-2.41(\mathrm{~m}, 1 \mathrm{H}), 2.28-2.32$ (m, 1H). ${ }^{13} \mathrm{C}$ NMR: $\delta 230.4,227.9,212.4,146.8,144.9,140.1,136.7,136.0,134.7,130.0,105.9$, 105.72, 105.71, 71.1, 67.8, 57.5, 56.3, 54.9, 54.8, 39.4, 28.9. HRMS (FAB) calcd for $\mathrm{C}_{21} \mathrm{H}_{23} \mathrm{BMoN}_{6} \mathrm{O}_{5}\left(\mathrm{M}^{+}\right)$: 548.0877. Found: 548.0867.

\section{( \pm )-Dicarbonyl[hydridotris(1-pyrazolyl)borato][(1S,2S,5R,6R,7S)-( $\left.\eta^{3}-2,3,4\right)-6$-acetyl-2-} methoxy-7-phenyl-8-oxabicyclo[3.2.1]oct-3-en-2-yl]molybdenum [( \pm -9-exo]

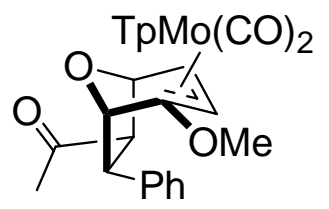

Following the general procedure, to a solution of the molybdenum complex 4 (126 mg, $0.207 \mathrm{mmol})$ in $\mathrm{CH}_{2} \mathrm{Cl}_{2}(10 \mathrm{~mL})$ was added solid sodium methoxide (34 $\left.\mathrm{mg}, 0.62 \mathrm{mmol}\right)$. The reaction mixture was stirred at room temperature for $24 \mathrm{~h} . \mathrm{Me}_{3} \mathrm{OBF}_{4}(77 \mathrm{mg}, 0.518 \mathrm{mmol})$ was added as a solid and the mixture was then stirred at room temperature for $2 \mathrm{~h}$. Purification by flash 
chromatography afforded the molybdenum complex ( \pm )-9-exo $(92 \mathrm{mg}, 71 \%)$. ( \pm )-9-exo: TLC $\left(\mathrm{R}_{f}=\right.$ 0.66, 1:1 hexanes-EtOAc). IR ( $\left.\mathrm{cm}^{-1}\right): 3127$ (w), 2949 (w), 2482 (w), 1992 (s), 1837 (s), 1710 (m), 1502 (m), 1409 (m), 1305 (m), 1212 (s), 1123 (m), 1050 (s). ${ }^{1} \mathrm{H}$ NMR: $\delta 8.42$ (d, J = $\left.1.91 \mathrm{~Hz}, 1 \mathrm{H}\right)$, $7.71(\mathrm{~d}, J=1.91 \mathrm{~Hz}, 1 \mathrm{H}), 7.63(\mathrm{~d}, J=1.91 \mathrm{~Hz}, 1 \mathrm{H}), 7.60$ (d, $J=1.91 \mathrm{~Hz}, 1 \mathrm{H}), 7.58$ (bs, 1H), 7.57 (bs, 2H), 7.47 (d, $J=1.91 \mathrm{~Hz}, 1 \mathrm{H}), 7.36(\mathrm{t}, J=7.62 \mathrm{~Hz}, 2 \mathrm{H}), 7.27$ (t, $J=7.30 \mathrm{~Hz}, 1 \mathrm{H}), 6.25(\mathrm{t}, J=$ $1.91 \mathrm{~Hz}, 1 \mathrm{H}), 6.22(\mathrm{t}, J=1.91 \mathrm{~Hz}, 1 \mathrm{H}), 6.05(\mathrm{t}, J=1.91 \mathrm{~Hz}, 1 \mathrm{H}), 4.70(\mathrm{~d}, J=5.72 \mathrm{~Hz}, 1 \mathrm{H}), 4.52(\mathrm{~d}$, $J=2.38 \mathrm{~Hz}, 1 \mathrm{H}), 4.23(\mathrm{t}, J=6.19 \mathrm{~Hz}, 1 \mathrm{H}), 3.90(\mathrm{dd}, J=7.63 \mathrm{~Hz}, J=2.86 \mathrm{~Hz}, 1 \mathrm{H}), 3.82(\mathrm{~d}, J=$ $6.67 \mathrm{~Hz}, 1 \mathrm{H}), 3.46(\mathrm{~d}, J=7.62 \mathrm{~Hz}, 1 \mathrm{H}), 2.23(\mathrm{~s}, 3 \mathrm{H}), 2.07(\mathrm{~s}, 3 \mathrm{H}) .{ }^{13} \mathrm{C}$ NMR: $\delta 230.5,228.4,206.7$, $146.4,145.1$, 139.9, 136.6, 136.2, 136.1, 134.9, 134.5, 129.0, 128.5, 127.5, 105.7, 105.6, 105.5, 81.0, 61.3, 59.0, 53.6, 53.48, 53.46, 28.3. HRMS (FAB) calcd for $\mathrm{C}_{27} \mathrm{H}_{27} \mathrm{BMoN}_{6} \mathrm{O}_{5}\left(\mathrm{M}^{+}\right): 624.1190$. Found: 624.1205.

( \pm )-Dicarbonyl[hydridotris(1-pyrazolyl)borato][(1R,2R,7S,8S,9S)-( $\left.\eta^{3}-9,10,11\right)-9-m e t h o x y-3-$ oxo-12-oxatricyclo[6.3.1.0 $\left.{ }^{2,7}\right]$ dodec-10-en-9-yl]molybdenum $[( \pm)-10]$, $(+)-$ dicarbonyl[hydridotris(1-pyrazolyl)borato][(1R,2R,7S,8S,9S)-( $\left.\eta^{3}-9,10,11\right)-9$-methoxy-3-oxo12-oxatricyclo[6.3.1.0 $\left.{ }^{2,7}\right]$ dodec-10-en-9-yl]molybdenum $\quad[(+)-10] \quad$ and $\quad(-)-$ dicarbonyl[hydridotris(1-pyrazolyl)borato][(1S,2S,7R,8R,9R)-( $\left.\eta^{3}-9,10,11\right)-9$-methoxy-3-oxo12-oxatricyclo[6.3.1.0 $\left.{ }^{2,7}\right]$ dodec-10-en-9-yl]molybdenum [(-)-10].

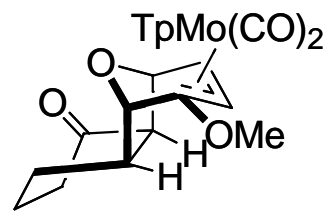

Following the general procedure, to a solution of the molybdenum complex 5 (600 $\mathrm{mg}, 1.08$ $\mathrm{mmol})$ in $\mathrm{CH}_{2} \mathrm{Cl}_{2}(45 \mathrm{~mL})$ was added solid sodium methoxide $(291 \mathrm{mg}, 5.39 \mathrm{mmol})$. The reaction mixture was stirred at room temperature for $20 \mathrm{~h} . \mathrm{Me}_{3} \mathrm{OBF}_{4}(639 \mathrm{mg}, 4.32 \mathrm{mmol})$ was added as a solid and the mixture was then stirred at room temperature for $1 \mathrm{~h}$. Purification by flash chromatography afforded the molybdenum complex $( \pm)-10(557 \mathrm{mg}, 90 \%) .( \pm)-10:$ TLC $\left(\mathrm{R}_{f}=0.50\right.$, 1:1 hexanes-EtOAc). IR ( $\left.\mathrm{cm}^{-1}\right): 3127$ (w), 3007 (m), 2482 (w), 1926 (s), 1837 (s), 1698 (m), 1409 (m), 1305 (m), 1278 (s), 1212 (s), 1123 (m), 1050 (s). ${ }^{1} \mathrm{H}$ NMR: $\delta 8.48$ (d, $\left.J=1.91 \mathrm{~Hz}, 1 \mathrm{H}\right), 7.95$ $(\mathrm{d}, J=1.91 \mathrm{~Hz}, 1 \mathrm{H}), 7.61(\mathrm{~d}, J=1.91 \mathrm{~Hz}, 2 \mathrm{H}), 7.59$ (d, $J=1.91 \mathrm{~Hz}, 1 \mathrm{H}), 7.50$ (d, $J=2.38 \mathrm{~Hz}, 1 \mathrm{H})$, $6.25(\mathrm{t}, J=1.91 \mathrm{~Hz}, 1 \mathrm{H}), 6.21(\mathrm{t}, J=1.91 \mathrm{~Hz}, 1 \mathrm{H}), 6.16(\mathrm{t}, J=1.91 \mathrm{~Hz}, 1 \mathrm{H}), 4.55(\mathrm{~d}, J=2.86 \mathrm{~Hz}$, 1H), $4.13(\mathrm{~s}, 1 \mathrm{H}), 3.91(\mathrm{dd}, J=7.62 \mathrm{~Hz}, J=3.33 \mathrm{~Hz}, 1 \mathrm{H}), 3.49$ (d, $J=7.62 \mathrm{~Hz}, 1 \mathrm{H}), 3.27$ (s, 3H), $3.14(\mathrm{~d}, J=9.05 \mathrm{~Hz}, 1 \mathrm{H}), 2.92(\mathrm{ddd}, J=15.25 \mathrm{~Hz}, J=9.53 \mathrm{~Hz}, J=6.20 \mathrm{~Hz}, 1 \mathrm{H}), 2.41(\mathrm{dt}, J=$ $18.10 \mathrm{~Hz}, J=5.24 \mathrm{~Hz}, 1 \mathrm{H}), 2.27(\mathrm{ddd}, J=16.67 \mathrm{~Hz}, J=9.53 \mathrm{~Hz}, J=7.62 \mathrm{~Hz}, 1 \mathrm{H}), 2.03-2.06(\mathrm{~m}$, 
$1 \mathrm{H}), 1.95-2.01(\mathrm{~m}, 1 \mathrm{H}), 1.68-1.76(\mathrm{~m}, 1 \mathrm{H}), 1.45-1.53(\mathrm{~m}, 1 \mathrm{H}) .{ }^{13} \mathrm{C}$ NMR: $\delta 228.8,228.2,212.2$, $146.5,144.7,140.5,136.5,136.0,134.5,132.4,105.8,105.6,105.5,81.4,79.2,61.1,58.1,56.5$, 52.7, 47.4, 38.9, 29.1, 20.9. HRMS (FAB) calcd for $\mathrm{C}_{23} \mathrm{H}_{25} \mathrm{BMoN}_{6} \mathrm{O}_{5}\left(\mathrm{M}^{+}\right)$: 574.1034. Found: 574.1011.

Following the general procedure, to a solution of the molybdenum complex 5 (112 $\mathrm{mg}, 0.20$ $\mathrm{mmol})$ in $\mathrm{CH}_{2} \mathrm{Cl}_{2}(10 \mathrm{~mL})$ was added solid potassium trimethylsilanonate $(77 \mathrm{mg}, 0.60 \mathrm{mmol})$. The reaction mixture was stirred at room temperature for $24 \mathrm{~h} . \mathrm{Me}_{3} \mathrm{OBF}_{4}(74 \mathrm{mg}, 0.50 \mathrm{mmol})$ was added as a solid and the mixture was then stirred at room temperature for $4 \mathrm{~h}$. Purification by flash chromatography afforded the molybdenum complex $( \pm)-10(111 \mathrm{mg}, 96.5 \%)$, exclusively.

Similar treatment of the chiral non-racemic (99.3\% ee) molybdenum complex (-)-5 (100 mg, $0.65 \mathrm{mmol}$ ) afforded (+)-10 as an orange solid (96 $\mathrm{mg}, 94 \%$ ) in $99.3 \%$ ee. The ee could be increased to $>99.9 \%$ by washing the product with acetonitrile $\left([\alpha]_{\mathrm{D}}=+372^{\circ}, c 1.95, \mathrm{CH}_{2} \mathrm{Cl}_{2}\right)$

Similar treatment of the chiral non-racemic $(99.3 \%$ ee) molybdenum complex $(+)-5(439 \mathrm{mg}$, $0.79 \mathrm{mmol}$ ) afforded (-)-10 as an orange solid (431 $\mathrm{mg}, 97.6 \%)$ in $99.3 \%$ ee. The ee could be increased to $>99.9 \%$ by washing the product with acetonitrile $\left([\alpha]_{\mathrm{D}}=-368^{\circ}, c 2.44, \mathrm{CH}_{2} \mathrm{Cl}_{2}\right)$.

\section{(土)-Dicarbonyl[hydridotris(1-pyrazolyl)borato][(1S,2S,3S,6R,7S)-( $\left.\eta^{3}-3,4,5\right)-3$-methoxy-8-oxo-}

\section{2-oxatricyclo[5.2.2.1 $\left.{ }^{2,6}\right]$ dodec-4-en-3-yl]molybdenum [( \pm )-15].}

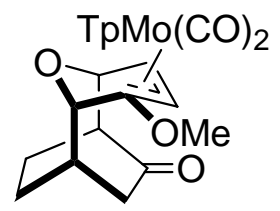

Following the general procedure, to a solution of the molybdenum complex 5 (320 $\mathrm{mg}, 0.58$ mmol) in $\mathrm{CH}_{2} \mathrm{Cl}_{2}(25 \mathrm{~mL})$ was added solid potassium tert-butoxide (195 mg, $\left.1.74 \mathrm{mmol}\right)$. The reaction mixture was stirred at room temperature for $20 \mathrm{~h} . \mathrm{Me}_{3} \mathrm{OBF}_{4}(215 \mathrm{mg}, 1.45 \mathrm{mmol})$ was added as a solid and the mixture was then stirred at room temperature for $4 \mathrm{~h}$. Purification by flash chromatography afforded an inseparable isomeric mixture of $( \pm)-\mathbf{1 0}$ and $( \pm)-\mathbf{1 5}(239 \mathrm{mg}, 73 \%)$. The ${ }^{1} \mathrm{H}$ NMR spectrum of the inseparable mixture showed a 1:2 ratio of 10:15. The pure isomer 15 was obtained by recrystallization from a mixture of dichloromethane and hexanes. $( \pm)$-15: TLC ( $\mathrm{R}_{f}$ $=0.50,1: 1$ hexanes-EtOAc). IR $\left(\mathrm{cm}^{-1}\right): 3127(\mathrm{w}), 2941(\mathrm{w}), 2482(\mathrm{w}), 1922(\mathrm{~s}), 1833$ (s), $1714(\mathrm{~s})$, 1505 (m), 1409 (s), 1305 (s), 1212 (s), 1123 (s), 1050 (s). ${ }^{1} \mathrm{H}$ NMR: $\delta 8.44$ (s, 1H), 7.86 (s, 1H), $7.62(\mathrm{~d}, J=1.91 \mathrm{~Hz}, 1 \mathrm{H}), 7.58(\mathrm{~d}, J=1.43 \mathrm{~Hz}, 1 \mathrm{H}), 7.50(\mathrm{~d}, J=2.38 \mathrm{~Hz}, 1 \mathrm{H}), 7.10(\mathrm{~d}, J=2.38 \mathrm{~Hz}$, 1H), 6.24(t, $J=1.91 \mathrm{~Hz}, 1 \mathrm{H}), 6.20(\mathrm{t}, J=1.91 \mathrm{~Hz}, 1 \mathrm{H}), 6.16(\mathrm{t}, J=1.91 \mathrm{~Hz}, 1 \mathrm{H}), 4.42(\mathrm{~d}, J=7.15$ Hz, 1H), $3.92(\mathrm{~d}, J=6.19 \mathrm{~Hz}, 1 \mathrm{H}), 3.75(\mathrm{~s}, 2 \mathrm{H}), 3.03(\mathrm{~s}, 3 \mathrm{H}), 2.83$ (t, $J=5.14 \mathrm{~Hz}, 1 \mathrm{H}), 2.69$ (m, 
1H), $2.53(\mathrm{bd}, J=19.53 \mathrm{~Hz}, 1 \mathrm{H}), 2.29$ (dd, $J=19.53 \mathrm{~Hz}, J=3.81 \mathrm{~Hz}, 1 \mathrm{H}), 2.18-2.24$ (m, 2H), 1.761.84 (m, 2H). ${ }^{13} \mathrm{C}$ NMR: $\delta 230.4,228.9,215.6,146.7,144.9,140.5,136.6,136.0,134.6,131.6$, 105.8, 105.7, 73.7, 73.1, 57.9, 57.3, 56.4, 54.6, 41.7, 34.8, 24.0, 22.0. HRMS (FAB) calcd for $\mathrm{C}_{23} \mathrm{H}_{25} \mathrm{BMoN}_{6} \mathrm{O}_{5}\left(\mathrm{M}^{+}\right)$: 574.1034. Found: 574.1024.

( \pm )-Dicarbonyl[hydridotris(1-pyrazolyl)borato][(1R,2R,6S,7S,8S)-( $\left.\eta^{3}-8,9,10\right)-8-$ methoxy-3oxo-11-oxatricyclo[5.3.1.0 $\left.{ }^{2,6}\right]$ undec-9-en-8-yl]molybdenum [( \pm )-11].

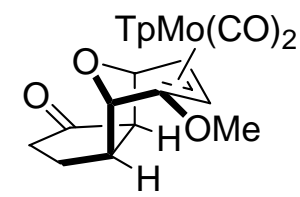

Following the general procedure, to a solution of the molybdenum complex 6 (108 $\mathrm{mg}, 0.2$ mmol) in $\mathrm{CH}_{2} \mathrm{Cl}_{2}(10 \mathrm{~mL})$ was added solid sodium methoxide $(54 \mathrm{mg}, 1.0 \mathrm{mmol})$. The reaction mixture was stirred at room temperature for $45 \mathrm{~h} . \mathrm{Me}_{3} \mathrm{OBF}_{4}(118 \mathrm{mg}, 0.80 \mathrm{mmol})$ was added as a solid and the mixture was then stirred at room temperature for $1 \mathrm{~h}$. Purification by flash chromatography afforded the molybdenum complex $( \pm)-11(557 \mathrm{mg}, 90 \%)$ exclusively. $( \pm)-11$ : TLC $\left(\mathrm{R}_{f}=\right.$ 0.53, 1:1 hexanes-EtOAc). IR $\left(\mathrm{cm}^{-1}\right): 3127(\mathrm{w}), 2968(\mathrm{w}), 2482(\mathrm{w}), 1926(\mathrm{~s}), 1841(\mathrm{~s})$, 1733 (s), 1515 (m), 1409 (s), 1305 (s), 1212 (s), 1123 (s), 1050 (s). ${ }^{1}$ H NMR: $\delta 8.46$ (d, J= 2.22 $\mathrm{Hz}, 1 \mathrm{H}), 7.95(\mathrm{~d}, J=1.91 \mathrm{~Hz}, 1 \mathrm{H}), 7.62$ (d, $J=1.91 \mathrm{~Hz}, 2 \mathrm{H}), 7.56(\mathrm{~d}, J=1.91 \mathrm{~Hz}, 1 \mathrm{H}), 7.50(\mathrm{~d}, J=$ $2.22 \mathrm{~Hz}, 1 \mathrm{H}), 6.25(\mathrm{t}, J=2.22 \mathrm{~Hz}, 1 \mathrm{H}), 6.22(\mathrm{t}, J=2.22 \mathrm{~Hz}, 1 \mathrm{H}), 6.17(\mathrm{t}, J=2.22 \mathrm{~Hz}, 1 \mathrm{H}), 4.38(\mathrm{~d}$, $J=2.86 \mathrm{~Hz}, 1 \mathrm{H}), 4.22(\mathrm{~d}, J=1.59 \mathrm{~Hz}, 1 \mathrm{H}), 3.84(\mathrm{dd}, J=7.62 \mathrm{~Hz}, J=2.86 \mathrm{~Hz}, 1 \mathrm{H}), 3.50(\mathrm{~d}, J=$ $5.94 \mathrm{~Hz}, 1 \mathrm{H}), 3.26$ (s, 3H), 3.19-3.23 (m, 1H), 3.06 (d, $J=7.62 \mathrm{~Hz}, 1 \mathrm{H}), 2.39-2.43(\mathrm{~m}, 1 \mathrm{H}), 2.23-$ $2.32(\mathrm{~m}, 2 \mathrm{H}), 1.85-1.89(\mathrm{~m}, 1 \mathrm{H}) .{ }^{13} \mathrm{C}$ NMR: $\delta 228.9,228.1,218.2,146.5,144.8,140.4,136.6$, 136.1, 134.5, 132.2, 105.9, 105.65, 105.58, 83.1, 79.8, 60.0, 59.1, 56.5, 53.2, 48.1, 39.4, 26.8. HRMS (FAB) calcd for $\mathrm{C}_{22} \mathrm{H}_{23} \mathrm{BMoN}_{6} \mathrm{O}_{5}\left(\mathrm{M}^{+}\right)$: 560.0877. Found: 560.0874.

\section{( \pm )-Dicarbonyl[hydridotris(1-pyrazolyl)borato][(1S,2S,5R,6R)-( $\left.\eta^{3}-2,3,4\right)-6$-acetyl-8-} benzyloxycarbonyl-2-methoxy-8-azabicyclo[3.2.1]oct-3-en-2-yl]molybdenum $[( \pm)-12-e x o]$ and ( \pm )-dicarbonyl[hydridotris(1-pyrazolyl)borato][(1S,2S,5R,6S)-( $\left.\eta^{3}-2,3,4\right)-6$-acetyl-8benzyloxycarbonyl-2-methoxy-8-azabicyclo[3.2.1]oct-3-en-2-yl]molybdenum [( \pm )-12-endo].
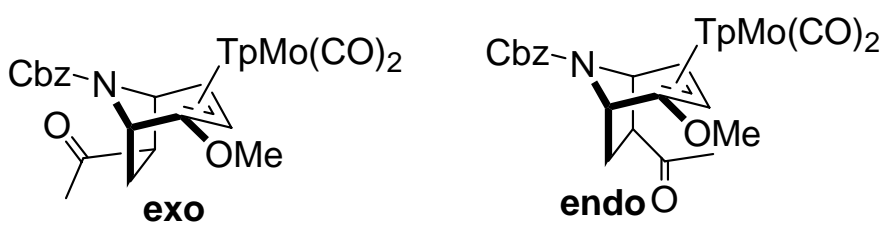
Following the general procedure, to a solution of the molybdenum complex 7 (840 mg, 1.24 mmol) in $\mathrm{CH}_{2} \mathrm{Cl}_{2}(85 \mathrm{~mL})$ was added solid sodium methoxide (402 $\mathrm{mg}, 7.44 \mathrm{mmol}$ ). The reaction mixture was stirred at room temperature for $6 \mathrm{~h} . \mathrm{Me}_{3} \mathrm{OBF}_{4}(918 \mathrm{mg}, 6.20 \mathrm{mmol})$ was added as a solid and the mixture was then stirred at room temperature for $15 \mathrm{~h}$. Purification by flash chromatography afforded the molybdenum complexes $( \pm)$-12-exo (760 mg, 79\%) and ( \pm )-12-endo (105 mg, 11\%). ( \pm )-12-exo: TLC $\left(\mathrm{R}_{f}=0.49,1: 1\right.$ hexanes-EtOAc). IR (cm $\left.{ }^{-1}\right): 3127(\mathrm{w}), 2945(\mathrm{w})$, 2482 (m), 1930 (s), 1841 (s), 1710 (s), 1502 (m), 1409 (s), 1305 (s), 1212 (s), 1116 (s), 1050 (s). ${ }^{1} \mathrm{H}$ NMR (a mixture of two rotamers): $\delta 8.42(\mathrm{~d}, J=1.59 \mathrm{~Hz}, 0.4 \mathrm{H}), 8.39$ (d, $J=1.59 \mathrm{~Hz}, 0.6 \mathrm{H}), 7.97$ $(\mathrm{d}, J=1.90 \mathrm{~Hz}, 0.6 \mathrm{H}), 7.95(\mathrm{~d}, J=1.59 \mathrm{~Hz}, 0.4 \mathrm{H}), 7.63$ (d, $J=1.59 \mathrm{~Hz}, 0.4 \mathrm{H}), 7.60(\mathrm{~m}, 2.6 \mathrm{H}), 7.47$ $(\mathrm{d}, J=2.22 \mathrm{~Hz}, 1 \mathrm{H}), 7.27-7.34(\mathrm{~m}, 5 \mathrm{H}), 6.23(\mathrm{t}, J=2.22 \mathrm{~Hz}, 1.2 \mathrm{H}), 6.18-6.21(\mathrm{~m}, 0.8 \mathrm{H}), 6.14-6,15$ $(\mathrm{m}, 1 \mathrm{H}), 5.08$ (AB quartet, $J=12.39 \mathrm{~Hz}, 0.8 \mathrm{H}), 5.03$ (AB quartet, $J=12.07 \mathrm{~Hz}, 1.2 \mathrm{H}), 4.77(\mathrm{~d}, J=$ $6.03 \mathrm{~Hz}, 0.6 \mathrm{H}), 4.69$ (d, $J=3.82 \mathrm{~Hz}, 0.4 \mathrm{H}), 4.65$ (d, $J=5.82 \mathrm{~Hz}, 0.4 \mathrm{H}), 4.57$ (d, J=4.13 Hz, 0.6H), $4.11(\mathrm{dd}, J=7.62 \mathrm{~Hz}, J=4.13 \mathrm{~Hz}, 0.4 \mathrm{H}), 4.03(\mathrm{dd}, J=7.62, J=4.13 \mathrm{~Hz}, 0.6 \mathrm{H}), 3.39-3.41(\mathrm{~m}, 1 \mathrm{H})$, 3.33-3.36 (m, 1H), $3.30(\mathrm{~s}, 1.8 \mathrm{H}), 3.22(\mathrm{~s}, 1.2 \mathrm{H}), 2.55-2.61(\mathrm{~m}, 1 \mathrm{H}), 2.31(\mathrm{~s}, 1.2 \mathrm{H}), 2.12(\mathrm{~s}, 1.8 \mathrm{H})$, $2.05(\mathrm{dd}, J=12.39 \mathrm{~Hz}, J=7.94 \mathrm{~Hz}, 0.6 \mathrm{H}), 1.98(\mathrm{dd}, J=12.86, J=8.10 \mathrm{~Hz}, 0.4 \mathrm{H}) .{ }^{13} \mathrm{C} \mathrm{NMR:} \delta$ 228.6, 228.3, 227.6, 227.5, 206.0, 205.9, 153.65, 153.56, 146.4, 144.8, 140.68, 140.60, 136.8, $136.6,136.5,136.1,135.70,135.66,134.5,128.5,128.4,128.3,128.1,127.9,105.8,105.6,67.4$, $62.8,62.4,59.5,59.3,59.14,59.13,58.5,56.8,56.6,52.7,52.4,33.9,33.7,29.2,29.1$. HRMS (FAB) calcd for $\mathrm{C}_{29} \mathrm{H}_{30} \mathrm{BMoN}_{7} \mathrm{O}_{6}\left(\mathrm{M}^{+}\right)$: 681.1405. Found: 681.1415. ( \pm )-12-endo: $\mathrm{TLC}\left(\mathrm{R}_{f}=0.69\right.$, 1:1 hexanes-EtOAc). IR ( $\left.\mathrm{cm}^{-1}\right): 3127$ (w), 2918 (m), 2482 (m), 1930 (s), 1845 (s), 1702 (s), 1502 (m), 1409 (s), 1366 (m), 1212 (s), 1050 (s). ${ }^{1} \mathrm{H}$ NMR (a mixture of two rotamers): $\delta$ 8.42, 8.40 (s, s, 1H), 7.97 (s, 1H), 7.61, 7.60 (s, s, 2H), 7.49 (d, J=1.91 Hz, 1H), 7.46 (d, J = 1.91 Hz, 1H), 7.32$7.44(\mathrm{~m}, 6 \mathrm{H}), 6.22(\mathrm{~s}, 1 \mathrm{H}), 6.19(\mathrm{~s}, 1 \mathrm{H}), 6.15(\mathrm{t}, J=1.90 \mathrm{~Hz}, 1 \mathrm{H}), 5.18$ (AB quartet, $J=12.39 \mathrm{~Hz}$, $0.8 \mathrm{H}), 5.15(\mathrm{AB}$ quartet, $J=12.38 \mathrm{~Hz}, 1.2 \mathrm{H}), 4.94(\mathrm{~s}, 0.6 \mathrm{H}), 4.87(\mathrm{~s}, 0.4 \mathrm{H}), 4.67(\mathrm{~d}, J=5.24 \mathrm{~Hz}$, $0.4 \mathrm{H}), 4.58(\mathrm{~d}, J=5.24 \mathrm{~Hz}, 0.6 \mathrm{H}), 3.71(\mathrm{dd}, J=7.63 \mathrm{~Hz}, J=4.28 \mathrm{~Hz}, 1 \mathrm{H}), 3.44(\mathrm{~m}, 0.6 \mathrm{H}), 3.37$ (m, $1 \mathrm{H}), 3.31(\mathrm{~m}, 0.4 \mathrm{H}), 3.27(\mathrm{~s}, 1.2 \mathrm{H}), 3.23(\mathrm{~s}, 1.8 \mathrm{H}), 2.60(\mathrm{~m}, 1 \mathrm{H}), 2.38(\mathrm{~s}, 1.8 \mathrm{H}), 2.36(\mathrm{~s}, 1.2 \mathrm{H})$, $2.10(\mathrm{~m}, 0.4 \mathrm{H}), 1.94(\mathrm{~m}, 0.6 \mathrm{H}) .{ }^{13} \mathrm{C}$ NMR: $\delta$ 229.4, 229.0, 227.7, 227.4, 205.32, 205.31, 153.9, $153.4,146.2,144.8,140.3,140.2,136.6,136.5,136.1,135.6,135.4,134.5,128.5,128.1,105.7$, 105.6, 67.6, 59.3, 58.5, 58.2, 57.8, 57.1, 57.0, 56.9, 56.8, 56.0, 53.6, 53.4, 33.0, 32.6, 30.74, 30.67, 29.8. HRMS (FAB) calcd for $\mathrm{C}_{29} \mathrm{H}_{30} \mathrm{BMoN}_{7} \mathrm{O}_{6}\left(\mathrm{M}^{+}\right)$: 681.1405. Found: 681.1418.

Following the general procedure, to a solution of the molybdenum complex 7 (100 mg, 0.15 mmol) in $\mathrm{CH}_{2} \mathrm{Cl}_{2}(7.5 \mathrm{~mL})$ was added solid potassium trimethylsilanolate $(57.9 \mathrm{mg}, 0.45 \mathrm{mmol})$. The reaction mixture was stirred at room temperature for $1 \mathrm{~h} . \mathrm{Me}_{3} \mathrm{OBF}_{4}(55.5 \mathrm{mg}, 0.723 \mathrm{mmol})$ 
was added as a solids and the mixture was then stirred at room temperature for $40 \mathrm{~min}$. Purification by flash chromatography afforded the molybdenum complexes $( \pm)-12-e x o ~(97 \mathrm{mg}, 95 \%)$ and $( \pm)$ 12-endo $(2 \mathrm{mg}, 2 \%)$. The diastereoselectivity is strongly related to the trapping time with the Meerwein salt. HPLC analysis of the reaction mixture indicated an exo / endo ratio of 17:1 in $4 \mathrm{~h}$ after addition of Meerwein salts. Prolongation of the reaction time to $15 \mathrm{~h}$ resulted in an exo / endo ratio of $5: 1$.

\section{( \pm )-Dicarbonyl[hydridotris(1-pyrazolyl)borato][(1R,6S,7S)-( $\left.\eta^{3}-7,8,9\right)-8$-benzyloxycarbonyl-7-} methoxy-3-oxo-10-azabicyclo[4.3.1]dec-8-en-7-yl]molybdenum [( \pm )-14].

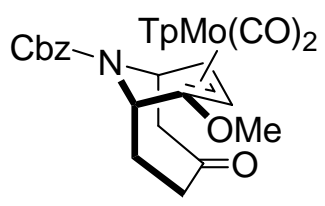

Following the general procedure, to a solution of the molybdenum complex 7 (100 mg, 0.15 mmol) in $\mathrm{CH}_{2} \mathrm{Cl}_{2}(10 \mathrm{~mL})$ was added solid potassium tert-butoxide $(50.4 \mathrm{mg}, 0.45 \mathrm{mmol})$. The reaction mixture was stirred at room temperature for $1 \mathrm{~h} . \mathrm{Me}_{3} \mathrm{OBF}_{4}(67 \mathrm{mg}, 0.45 \mathrm{mmol})$ was added as a solids and the mixture was then stirred at room temperature for $4 \mathrm{~h}$. Purification by flash chromatography afforded the molybdenum complexes $( \pm)$-12-exo (23 mg, 23\%), ( \pm )-12-endo (3 mg, $3 \%)$ and $( \pm)-14(53 \mathrm{mg}, 52 \%) .14$ was often contaminated by small amount of the starting material 7. However, the pure isomer $\mathbf{1 4}$ was obtained by recrystallization from a mixture of dichloromethane and hexanes. $( \pm)-14$ : TLC $\left(\mathrm{R}_{f}=0.58,1: 1\right.$ hexanes-EtOAc). IR $\left(\mathrm{cm}^{-1}\right): 3127(\mathrm{w})$, 2957 (w), 2934 (w), 2482 (m), 1930 (s), 1841 (s), 1968 (s), 1505 (m), 1474 (s), 1305 (s), 1216 (s), 1119 (s), 1050 (s). ${ }^{1} \mathrm{H}$ NMR (a mixture of two rotamers): $\delta 8.40$ (d, $\left.J=2.22 \mathrm{~Hz}, 0.5 \mathrm{H}\right), 8.39$ (d, $J=$ $2.22 \mathrm{~Hz}, 0.5 \mathrm{H}), 7.87(\mathrm{~d}, J=2.22 \mathrm{~Hz}, 0.5 \mathrm{H}), 7.83(\mathrm{~d}, J=2.22 \mathrm{~Hz}, 0.5 \mathrm{H}), 7.62(\mathrm{~m}, 2 \mathrm{H}), 7.59(\mathrm{~m}$, 1H), 7.49 (t, $J=2.22 \mathrm{~Hz}, 1 \mathrm{H}), 7.33-7.40(\mathrm{~m}, 5 \mathrm{H}), 6.22-6.25(\mathrm{~m}, 2 \mathrm{H}), 6.16-6.18(\mathrm{~m}, 1 \mathrm{H}), 5.20(\mathrm{~s}$, $1 \mathrm{H}), 5.18$ (AB quartet, $J=12.39 \mathrm{~Hz}, 1 \mathrm{H}), 5.00(\mathrm{t}, J=3.81 \mathrm{~Hz}, 0.5 \mathrm{H}), 4.96(\mathrm{t}, J=3.81 \mathrm{~Hz}, 0.5 \mathrm{H})$, $4.67(\mathrm{~m}, 0.5 \mathrm{H}), 4.58(\mathrm{~m}, 0.5 \mathrm{H}), 3.89(\mathrm{dd}, J=7.94 \mathrm{~Hz}, J=2.54 \mathrm{~Hz}, 0.5 \mathrm{H}), 3.83(\mathrm{dd}, J=8.26, J=$ $2.54 \mathrm{~Hz}, 0.5 \mathrm{H}), 3.77(\mathrm{t}, J=8.10 \mathrm{~Hz}, 1 \mathrm{H}), 3.19(\mathrm{dd}, J=13.65 \mathrm{~Hz}, J=4.76 \mathrm{~Hz}, 0.5 \mathrm{H}), 3.05(\mathrm{dd}, J=$ $13.65 \mathrm{~Hz}, J=4.45 \mathrm{~Hz}, 0.5 \mathrm{H}), 3.03(\mathrm{~s}, 1.5 \mathrm{H}), 2.96(\mathrm{~s}, 1.5 \mathrm{H}), 2.89(\mathrm{dd}, J=11.12 \mathrm{~Hz}, J=3.18 \mathrm{~Hz}$, $0.5 \mathrm{H}), 2.86(\mathrm{dd}, J=11.76 \mathrm{~Hz}, J=3.50 \mathrm{~Hz}, 0.5 \mathrm{H}), 2.48-2.69(\mathrm{~m}, 2 \mathrm{H}), 2.01-2.26(\mathrm{~m}, 2 \mathrm{H}) .{ }^{13} \mathrm{C}$ NMR: $\delta 230.1,229.7,227.5,227.2,211.2,154.2,153.7,146.8,145.03,144.99,140.4,140.2,136.7,136.1$, 134.7, 128.6, 128.4, 128.25, 128.19, 127.9, 105.9, 105.8, 105.7, 67.9, 67.8, 58.0, 57.8, 57.6, 56.9, 55.14, 55.09, 54.0, 53.6, 53.1, 47.6, 47.4, 39.9, 39.8, 27.8, 27.0. HRMS (FAB) calcd for $\mathrm{C}_{29} \mathrm{H}_{31} \mathrm{BMoN}_{7} \mathrm{O}_{6}\left([\mathrm{M}+\mathrm{H}]^{+}\right): 682.1483$. Found: 682.1515 . 


\section{DEMETALLATION OF 1,5-MICHAEL-TYPE ADDUCTS}

To an orange solution of the adducts $( \pm)-\mathbf{1 0},(+)-\mathbf{1 0},(-)-\mathbf{1 0}$ and $( \pm)-\mathbf{1 2 - e x o}(1.0 \mathrm{mmol})$ and triethylamine $(1.5 \mathrm{mmol})$ in a $3: 1$ mixture of $\mathrm{THF} / \mathrm{H}_{2} \mathrm{O}(50 \mathrm{~mL})$ at $0{ }^{\circ} \mathrm{C}$ (ice bath) open to air was added a solution of ceric ammonium nitrate $(8.0 \mathrm{mmol})$ in $\mathrm{H}_{2} \mathrm{O}(25 \mathrm{~mL})$ drop-wise over $5 \mathrm{~min}$. After completion of the addition the color faded and a light yellow solution was formed. The ice bath was removed and the reaction mixtures were stirred for an additional $10 \mathrm{~min}$ at room temperature and then partitioned between dichloromethane $(40 \mathrm{~mL})$ and water $(40 \mathrm{~mL})$. The organic layers were washed with brine, dried with $\mathrm{Na}_{2} \mathrm{SO}_{4}$, and solvents were removed under vacuum to provide the crude products. The crude products were purified by flash chromatography to afford $( \pm)-\mathbf{1 6},(+)-\mathbf{1 6},(-)-16$ and $( \pm)-\mathbf{1 7}$, as colorless crystalline solids or oils.

\section{$( \pm)-(1 S, 2 R, 7 S, 8 S)-3-O x o-12-0 x a t r i c y c l o\left[6.3 .1 .0^{2,7}\right]$ dodec-10-en-9-one $[( \pm)-16],(+)-$ $(1 R, 2 S, 7 R, 8 R)-3-0 x 0-12-0 x a t r i c y c l o\left[6.3 .1 .0^{2,7}\right]$ dodec-10-en-9-one $[(+)-16]$ and (-)- $(1 S, 2 R, 7 S, 8 S)-3-0 x 0-12-0 x a t r i c y c l o\left[6.3 .1 .0^{2,7}\right]$ dodec-10-en-9-one [(-)-16].

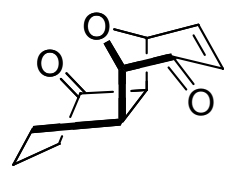

The molybdenum complex $( \pm)-10(340 \mathrm{mg}, 0.60 \mathrm{mmol})$ was treated with TEA $(126 \mu \mathrm{L}, 0.90$ mmol) and CAN (2.59 g, $4.8 \mathrm{mmol})$ according to the general procedure. Flash chromatography over silica gel eluting with a 1:1 EtOAc/hexanes mixture afforded the tricyclodione $( \pm)-16(84 \mathrm{mg}$, $87 \%)$ as a colorless solid. $( \pm)-16$ : $\operatorname{TLC}\left(\mathrm{R}_{f}=0.39,1: 1\right.$ hexanes-EtOAc); $\mathrm{mp}=76-77^{\circ} \mathrm{C} . \mathrm{IR}\left(\mathrm{cm}^{-1}\right)$ : 2949 (m), 2872 (m), 1695(s), 1455 (m), 1378 (m), 1278 (s). ${ }^{1} \mathrm{H}$ NMR: $\delta 7.31$ (dd, $J=9.85 \mathrm{~Hz}, J=$ $4.45 \mathrm{~Hz}, 1 \mathrm{H}), 6.04(\mathrm{dd}, J=9.85 \mathrm{~Hz}, J=1.28 \mathrm{~Hz}, 1 \mathrm{H}), 5.33$ (d, $J=4.45 \mathrm{~Hz}, 1 \mathrm{H}), 4.30$ (s, 1H), $2.70-$ $2.79(\mathrm{~m}, 2 \mathrm{H}), 2.43(\mathrm{t}, J=6.67 \mathrm{~Hz}, 2 \mathrm{H}), 2.18-2.25(\mathrm{~m}, 1 \mathrm{H}), 1.83-2.20(\mathrm{~m}, 2 \mathrm{H}), 1.53-1.61(\mathrm{~m}, 1 \mathrm{H})$. ${ }^{13} \mathrm{C}$ NMR: $\delta$ 209.2, 196.0, 151.9, 126.5, 88.0, 76.0, 53.2, 40.3, 38.8, 28.3, 20.4. HRMS (EI) calcd for $\mathrm{C}_{11} \mathrm{H}_{12} \mathrm{O}_{3}\left(\mathrm{M}^{+}\right)$: 192.0786. Found: 192.0787.

Similar treatment of (-)-10 $(220 \mathrm{mg}, 0.386 \mathrm{mmol})$ with TEA $(80.6 \mu \mathrm{L}, 0.579 \mathrm{mmol})$ and CAN (1.67 g, $3.09 \mathrm{mmol}$ ) according to the general procedure and subsequent flash chromatography afforded the tricyclodione $(+)-16(75.4 \mathrm{mg}, 97 \%)$ as a colorless solid in $>99.9 \%$ ee $\left([\alpha]_{\mathrm{D}}=+274^{\circ}\right.$, C $\left.1.84, \mathrm{CH}_{2} \mathrm{Cl}_{2}\right) . \mathrm{Mp}=83-84^{\circ} \mathrm{C}$. 
Similar treatment of $(+)-\mathbf{1 0}(114 \mathrm{mg}, 0.20 \mathrm{mmol})$ with TEA $(41.7 \mu \mathrm{L}, 0.30 \mathrm{mmol})$ and CAN (864 $\mathrm{mg}, 1.6 \mathrm{mmol}$ ) according to the general procedure and subsequent flash chromatography afforded the tricyclodione (-)-16 (35.6 mg, 93\%) as a colorless solid in $>99.9 \%$ ee $\left([\alpha]_{\mathrm{D}}=-270^{\circ}, c\right.$ 1.07, $\left.\mathrm{CH}_{2} \mathrm{Cl}_{2}\right) . \mathrm{Mp}=74-75^{\circ} \mathrm{C}$.

The molybdenum complex $( \pm)-\mathbf{1 0}(57 \mathrm{mg}, 0.10 \mathrm{mmol})$ was treated with $\mathrm{CuCl}_{2}(94.1 \mathrm{mg}$, $0.70 \mathrm{mmol}$ ) in a $2 \mathrm{~mL} \mathrm{CH}_{2} \mathrm{Cl}_{2}$ solution. The reaction mixture was stirred at room temperature for 2 h. The color of the solution changed from red-brown to green. Flash chromatography over silica gel eluting with a 1:1 EtOAc/hexanes mixture afforded the tricyclodione $( \pm)-16(14 \mathrm{mg}, 74 \%)$ as a colorless solid.

( \pm )-(1S,5S,6R)- 6-Acetyl-8-benzyloxycarbonyl-8-azabicyclo[3.2.1]oct-3-en-2-one $( \pm)-[( \pm)-17]$.

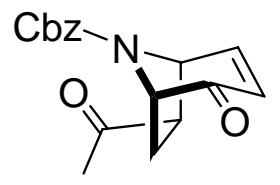

The molybdenum complex $( \pm)$-12-exo $(1.0 \mathrm{~g}, 1.47 \mathrm{mmol})$ was treated with TEA $(310 \mu \mathrm{L}$, $2.21 \mathrm{mmol})$ and $\mathrm{CAN}(6.45 \mathrm{~g}, 11.8 \mathrm{mmol})$ according to the general procedure. Flash chromatography over silica gel eluting with a 1:1 EtOAc/hexanes mixture afforded the tricyclodione $( \pm)-\mathbf{1 7}(0.38 \mathrm{~g}, 87 \%)$ as a colorless oil. $( \pm)-17$ : TLC $\left(\mathrm{R}_{f}=0.27,1: 1\right.$ hexanes-EtOAc). IR (cm $\left.{ }^{-1}\right): 3034$ (w), 2957 (w), 1702(s), 1409 (s), 1363 (s), 1312 (s), 1100(s). ${ }^{1}$ H NMR: $\delta$ 7.28-7.38 (m, 6H), $6.03(\mathrm{dd}, J=9.84 \mathrm{~Hz}, J=1.63 \mathrm{~Hz}, 1 \mathrm{H}), 5.11$ (AB quartet, $J=12.38 \mathrm{~Hz}, 2 \mathrm{H}), 5.06$ (br s, 1H), 4.80 (br s, 0.4H), 4.73(br s, 0.6H), 3.04 (dd, $J=9.21 \mathrm{~Hz}, J=3.81 \mathrm{~Hz}, 1 \mathrm{H}), 2.82$ (br s, 0.6H), 2.66 (br s, 0.4H), 2.29, 2,24 (br s, br s, 3.4H), 1.91 (br s, 1.6H). ${ }^{13} \mathrm{C}$ NMR: $\delta 204.7,204.5,195.5$, 195.1, 154.3, 154.1, 151.9, 150.9, 135.9, 128.7, 128.4, 128.15, 128.06, 67.9, 64.3, 56.5, 56.0, 54.8, 53.9, 29.9, 28.5, 27.5, 27.3. HRMS (FAB) calcd for $\mathrm{C}_{17} \mathrm{H}_{18} \mathrm{NO}_{4}\left([\mathrm{M}+\mathrm{H}]^{+}\right)$: 300.1236. Found: 300.1230 .

\section{DETERMINATION OF ENANTIOMERIC EXCESSES BY HPLC}

HPLC was performed on an Agilent 1100 Series HPLC chromatograph with ZORBAX Eclipse XDB-C8 $(4.6 \times 150 \mathrm{~mm}, 5 \mu \mathrm{m})$, CHIRALPAK AS-RH, CHIRALPAK OD-RH and CHIRALPAK AD-RH columns at room temperature using a Diode Array Detector (DAD) with multiple wavelengths $(210 \mathrm{~nm}, 230 \mathrm{~nm}, 254 \mathrm{~nm}$, and $280 \mathrm{~nm})$. Samples of products for HPLC analyses were prepared by dissolving several milligrams of pure compound in 2-3 $\mathrm{mL}$ of $\mathrm{CH}_{3} \mathrm{CN}$. 
One microliter $(1 \mu \mathrm{L})$ of the solution was injected for the HPLC analysis. HPLC grade acetonitrile (or acetonitrile containing $0.1 \%$ of trifluoroacetic acid) and water were used. Racemic samples were run first to find both enantiomers. The results are listed in Table S1.

Table S1. Determination of \% ee by HPLC ${ }^{b}$

\begin{tabular}{|c|c|c|c|c|}
\hline Entry & Compd & Column $^{\mathrm{a}}$ & $\begin{array}{c}\text { Retention time }^{\mathrm{c}} \\
\text { of }(-) \text { isomer }\end{array}$ & $\begin{array}{c}\text { Retention time }^{\mathrm{c}} \\
\text { of }(+) \text { isomer }\end{array}$ \\
\hline 1 & $\mathbf{1}$ & AS-RH & 9.5 & 8.8 \\
2 & $\mathbf{5}$ & AS-RH & 11.4 & 9.2 \\
3 & $\mathbf{1 0}$ & AS-RH & 10.5 & 9.8 \\
4 & $\mathbf{1 6}$ & AD-RH & 4.9 & 6.2 \\
\hline
\end{tabular}

${ }^{\mathrm{a}}$ CHIRALPAK AS-RH, CHIRALPAK AD-RH. ${ }^{\mathrm{b}}$ Gradient eluent except for 16: $40 \%$ of $\mathrm{CH}_{3} \mathrm{CN}$ in $\mathrm{H}_{2} \mathrm{O}(3 \mathrm{~min}), 65 \%$ of $\mathrm{CH}_{3} \mathrm{CN}$ in $\mathrm{H}_{2} \mathrm{O}(7.5 \mathrm{~min}), 99 \%$ of $\mathrm{CH}_{3} \mathrm{CN}$ in $\mathrm{H}_{2} \mathrm{O}(9 \mathrm{~min})$; for $16,30 \%$ of $\mathrm{CH}_{3} \mathrm{CN}, 70 \%$ of $\mathrm{CH}_{3} \mathrm{CN}$ containing $0.1 \%$ of $\mathrm{CF}_{3} \mathrm{COOH}$. Flowrate $1 \mathrm{~mL} / \mathrm{min}$. DAD detector $\lambda=$ $254 \mathrm{~nm}^{\mathrm{c}}{ }^{\mathrm{in}}$ minutes.

\section{X-RAY CRYSTALLOGRAPHIC STUDIES.}

Crystals of $( \pm)-\mathbf{1 3}$ were obtained by recrystallization from dichloromethane and hexanes. A suitable single crystal was coated with Paratone $\mathrm{N}$ oil, suspended in a small fiber loop and placed in a cooled nitrogen gas stream at $173 \mathrm{~K}$ on a Bruker D8 SMART APEX CCD sealed tube diffractometer with graphite monochromated $\mathrm{MoK}_{\alpha}(0.71073 \AA)$ radiation. Data were measured using a series of combinations of phi and omega scans with $10 \mathrm{~s}$ frame exposures and $0.3^{\circ}$ frame widths. Data collection, indexing and initial cell refinements were all carried out using SMART ${ }^{6}$ software. Frame integration and final cell refinements were done using $\mathrm{SAINT}^{7}$ software. The final cell parameters were determined from least-squares refinement on 5054 reflections. The SADABS ${ }^{8}$ program was used to carry out absorption corrections.

The structure was solved using Direct methods and difference Fourier techniques (SHELXTL, V6.12). ${ }^{9} \quad$ Hydrogen atoms were placed their expected chemical positions using the HFIX command and were included in the final cycles of least squares with isotropic $\mathrm{U}_{\mathrm{ij}}$ 's related to the atom's ridden upon. The $\mathrm{C}-\mathrm{H}$ distances were fixed at $0.93 \AA$ (aromatic and amide), $0.98 \AA$ (methine), $0.97 \AA$ (methylene), or $0.96 \AA$ (methyl). All non-hydrogen atoms were refined anisotropically. Scattering factors and anomalous dispersion corrections are taken from the 
International Tables for X-ray Crystallography. ${ }^{10}$ Structure solution, refinement, graphics and generation of publication materials were performed by using SHELXTL, V6.12 software. Additional details of data collection and structure refinement are given in Table S2.

Figure S1. ORTEP drawing of ( \pm -dicarbonyl[hydridotris(1pyrazolyl)borato][(1R,6S,7S)-( $\left.\eta^{3}-7,8,9\right)-7-$ methoxy-3-0xo-10-oxabicyclo[4.3.1]dec-8-en-7yl]molybdenum [( \pm -13].

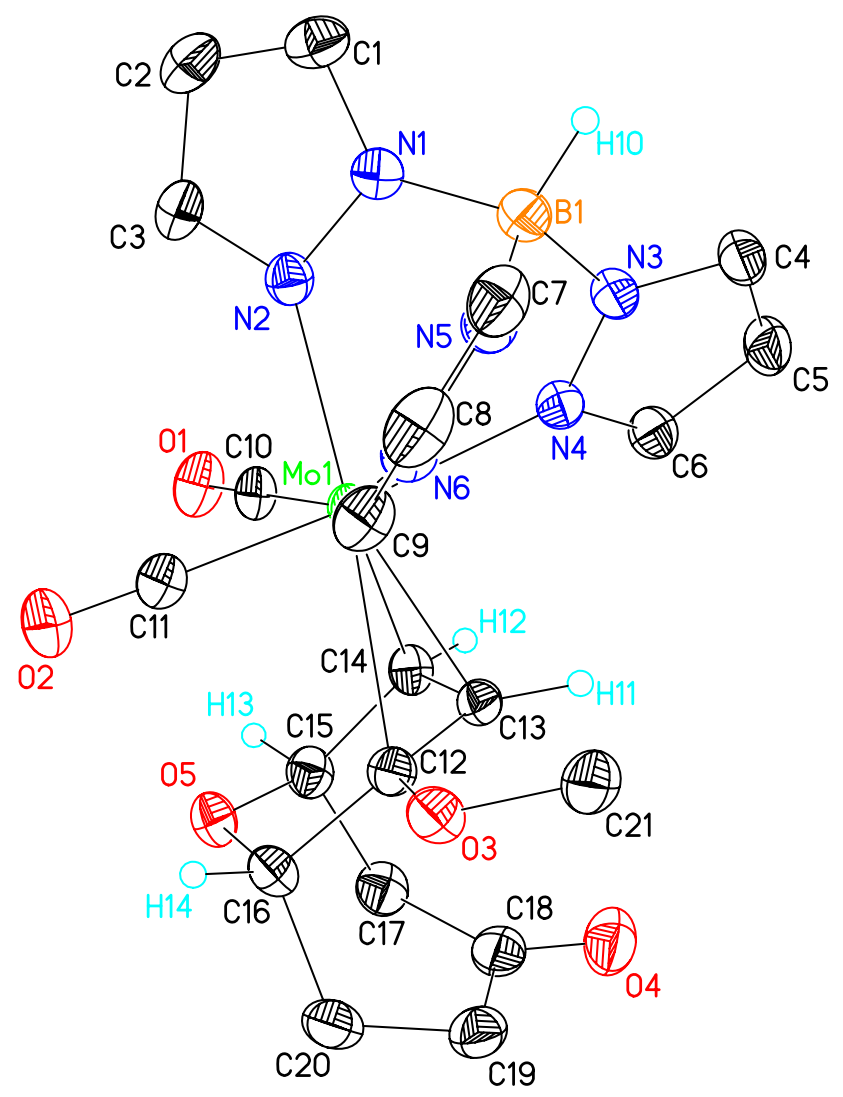

Table S2. Crystal data and structure refinement for $( \pm)-\mathbf{1 3}$.

Identification code

zyq26s

Empirical formula

$\mathrm{C}_{22} \mathrm{H}_{24} \mathrm{BC}_{13} \mathrm{MoN}_{6} \mathrm{O}$

Formula weight

665.57

Temperature

173(2) K

Wavelength

$0.71073 \AA$ 
Crystal system

Space group

Unit cell dimensions

Volume

Z

Density (calculated)

Absorption coefficient

$\mathrm{F}(000)$

Crystal size

Theta range for data collection

Index ranges

Reflections collected

Independent reflections

Completeness to theta $=28.34^{\circ}$

Absorption correction

Max. and min. transmission

Refinement method

Data / restraints / parameters

Goodness-of-fit on $\mathrm{F}^{2}$

Final $\mathrm{R}$ indices [I $>2 \operatorname{sigma}(\mathrm{I})]$

$\mathrm{R}$ indices (all data)

Largest diff. peak and hole
Triclinic

P-1

$$
\begin{array}{ll}
\mathrm{a}=8.7750(17) \AA & \alpha=89.165(4)^{\circ} . \\
\mathrm{b}=10.558(2) \AA & \beta=79.929(4)^{\circ} . \\
\mathrm{c}=15.442(3) \AA & \gamma=67.509(3)^{\circ} .
\end{array}
$$

1299.3(4) $\AA^{3}$

2

\section{$1.701 \mathrm{Mg} / \mathrm{m}^{3}$}

$0.860 \mathrm{~mm}^{-1}$

672

$0.29 \times 0.20 \times 0.12 \mathrm{~mm}^{3}$

2.09 to $28.34^{\circ}$.

$-11<=\mathrm{h}<=11,-14<=\mathrm{k}<=14,-20<=\mathrm{l}<=20$

18179

$6482[\mathrm{R}($ int $)=0.0254]$

$99.6 \%$

Semi-empirical from equivalents

0.9038 and 0.7885

Full-matrix least-squares on $\mathrm{F}^{2}$

6482 / 0 / 439

1.121

$\mathrm{R} 1=0.0306, \mathrm{wR} 2=0.0750$

$\mathrm{R} 1=0.0326, \mathrm{wR} 2=0.0762$

1.333 and -0.970 e. $\AA^{-3}$

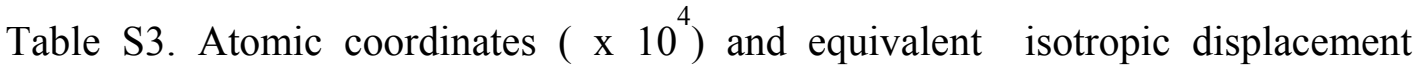


parameters $\left(\AA^{2} \times 10^{3}\right)$ for $( \pm)-13$. U(eq) is defined as one third of the trace of the orthogonalized $\mathrm{U}^{\mathrm{ij}}$ tensor.

\begin{tabular}{|c|c|c|c|c|}
\hline & $\mathrm{x}$ & $\mathrm{y}$ & z & $\mathrm{U}(\mathrm{eq})$ \\
\hline $\mathrm{B}(1)$ & $13529(3)$ & $5201(2)$ & $3069(2)$ & $26(1)$ \\
\hline$C(1)$ & $14578(3)$ & $6207(2)$ & $4272(2)$ & $29(1)$ \\
\hline$C(2)$ & $13958(3)$ & $7392(3)$ & $4803(2)$ & $33(1)$ \\
\hline$C(3)$ & $12418(3)$ & $8149(2)$ & $4578(1)$ & $27(1)$ \\
\hline$C(4)$ & $14568(3)$ & $5481(2)$ & $1418(2)$ & $30(1)$ \\
\hline$C(5)$ & $14060(3)$ & $6455(3)$ & $820(2)$ & $32(1)$ \\
\hline$C(6)$ & $12590(3)$ & $7458(2)$ & $1275(1)$ & $27(1)$ \\
\hline$C(7)$ & $11898(3)$ & $3672(2)$ & $3676(2)$ & $31(1)$ \\
\hline $\mathrm{C}(8)$ & $10255(3)$ & $3919(2)$ & $4021(2)$ & $33(1)$ \\
\hline $\mathrm{C}(9)$ & $9378(3)$ & $5294(2)$ & $3903(1)$ & $27(1)$ \\
\hline$C(10)$ & $9985(3)$ & $9870(2)$ & $3398(1)$ & $23(1)$ \\
\hline $\mathrm{C}(11)$ & $8236(3)$ & $8614(2)$ & $4279(1)$ & $23(1)$ \\
\hline$C(12)$ & $7333(2)$ & $8123(2)$ & $2741(1)$ & $22(1)$ \\
\hline$C(13)$ & $8589(2)$ & $8289(2)$ & $2124(1)$ & $20(1)$ \\
\hline$C(14)$ & $8775(3)$ & $9568(2)$ & $2240(1)$ & $22(1)$ \\
\hline$C(15)$ & $7171(3)$ & $10814(2)$ & $2542(1)$ & $25(1)$ \\
\hline$C(16)$ & $5759(2)$ & $9352(2)$ & $3129(1)$ & $24(1)$ \\
\hline$C(17)$ & $6265(3)$ & $11392(2)$ & $1760(2)$ & $29(1)$ \\
\hline$C(18)$ & $6104(3)$ & $10329(2)$ & $1182(2)$ & $31(1)$ \\
\hline$C(19)$ & $5052(3)$ & $9529(2)$ & $1549(2)$ & $32(1)$ \\
\hline$C(20)$ & $4426(3)$ & $9621(2)$ & $2544(2)$ & $31(1)$ \\
\hline$C(21)$ & $7949(3)$ & $5888(2)$ & $2114(2)$ & $31(1)$ \\
\hline$C(1 S)$ & $1253(3)$ & $2557(3)$ & $781(2)$ & $34(1)$ \\
\hline
\end{tabular}




$\begin{array}{lrrrr}\mathrm{Cl}(1 \mathrm{~S}) & 607(1) & 2117(1) & 1839(1) & 39(1) \\ \mathrm{Cl}(2 \mathrm{~S}) & -483(1) & 3343(1) & 260(1) & 51(1) \\ \mathrm{Cl}(3 \mathrm{~S}) & 2300(1) & 3675(1) & 867(1) & 54(1) \\ \mathrm{Mo}(1) & 9993(1) & 8047(1) & 3230(1) & 18(1) \\ \mathrm{N}(1) & 13464(2) & 6260(2) & 3765(1) & 24(1) \\ \mathrm{N}(2) & 12101(2) & 7473(2) & 3952(1) & 23(1) \\ \mathrm{N}(3) & 13455(2) & 5880(2) & 2179(1) & 24(1) \\ \mathrm{N}(4) & 12213(2) & 7113(2) & 2095(1) & 23(1) \\ \mathrm{N}(5) & 11988(2) & 4844(2) & 3353(1) & 25(1) \\ \mathrm{N}(6) & 10416(2) & 5859(2) & 3492(1) & 24(1) \\ \mathrm{O}(1) & 9958(2) & 10952(2) & 3542(1) & 31(1) \\ \mathrm{O}(2) & 7222(2) & 8918(2) & 4913(1) & 32(1) \\ \mathrm{O}(3) & 6967(2) & 6982(2) & 2766(1) & 26(1) \\ \mathrm{O}(4) & 6809(2) & 10125(2) & 413(1) & 41(1) \\ \mathrm{O}(5) & 6096(2) & 10549(1) & 3257(1) & 25(1)\end{array}$

Table S4. Bond lengths $[\AA]$ and angles $\left[{ }^{\circ}\right]$ for $( \pm)-13$.

$\begin{array}{ll}\mathrm{B}(1)-\mathrm{N}(5) & 1.530(3) \\ \mathrm{B}(1)-\mathrm{N}(1) & 1.540(3) \\ \mathrm{B}(1)-\mathrm{N}(3) & 1.541(3) \\ \mathrm{B}(1)-\mathrm{H}(10) & 1.05(2) \\ \mathrm{C}(1)-\mathrm{N}(1) & 1.341(3) \\ \mathrm{C}(1)-\mathrm{C}(2) & 1.374(3) \\ \mathrm{C}(1)-\mathrm{H}(1) & 0.88(3) \\ \mathrm{C}(2)-\mathrm{C}(3) & 1.384(3)\end{array}$




\begin{tabular}{|c|c|}
\hline $\mathrm{C}(2)-\mathrm{H}(2)$ & $0.90(3)$ \\
\hline $\mathrm{C}(3)-\mathrm{N}(2)$ & $1.337(3)$ \\
\hline $\mathrm{C}(3)-\mathrm{H}(3)$ & $0.90(3)$ \\
\hline $\mathrm{C}(4)-\mathrm{N}(3)$ & $1.346(3)$ \\
\hline$C(4)-C(5)$ & $1.368(3)$ \\
\hline $\mathrm{C}(4)-\mathrm{H}(4)$ & $0.90(3)$ \\
\hline$C(5)-C(6)$ & $1.392(3)$ \\
\hline $\mathrm{C}(5)-\mathrm{H}(5)$ & $0.89(3)$ \\
\hline $\mathrm{C}(6)-\mathrm{N}(4)$ & $1.333(3)$ \\
\hline $\mathrm{C}(6)-\mathrm{H}(6)$ & $0.89(2)$ \\
\hline $\mathrm{C}(7)-\mathrm{N}(5)$ & $1.353(3)$ \\
\hline$C(7)-C(8)$ & $1.370(4)$ \\
\hline $\mathrm{C}(7)-\mathrm{H}(7)$ & $0.89(3)$ \\
\hline $\mathrm{C}(8)-\mathrm{C}(9)$ & $1.386(3)$ \\
\hline $\mathrm{C}(8)-\mathrm{H}(8)$ & $0.85(3)$ \\
\hline $\mathrm{C}(9)-\mathrm{N}(6)$ & $1.338(3)$ \\
\hline $\mathrm{C}(9)-\mathrm{H}(9)$ & $0.93(2)$ \\
\hline $\mathrm{C}(10)-\mathrm{O}(1)$ & $1.158(2)$ \\
\hline $\mathrm{C}(10)-\mathrm{Mo}(1)$ & $1.943(2)$ \\
\hline $\mathrm{C}(11)-\mathrm{O}(2)$ & $1.158(2)$ \\
\hline $\mathrm{C}(11)-\mathrm{Mo}(1)$ & $1.951(2)$ \\
\hline $\mathrm{C}(12)-\mathrm{O}(3)$ & $1.358(2)$ \\
\hline$C(12)-C(13)$ & $1.389(3)$ \\
\hline$C(12)-C(16)$ & $1.521(3)$ \\
\hline $\mathrm{C}(12)-\mathrm{Mo}(1)$ & $2.549(2)$ \\
\hline$C(13)-C(14)$ & $1.438(3)$ \\
\hline $\mathrm{C}(13)-\mathrm{Mo}(1)$ & $2.2331(19)$ \\
\hline
\end{tabular}




\begin{tabular}{|c|c|}
\hline $\mathrm{C}(13)-\mathrm{H}(11)$ & $0.97(2)$ \\
\hline$C(14)-C(15)$ & $1.517(3)$ \\
\hline C(14)-Mo(1) & $2.291(2)$ \\
\hline $\mathrm{C}(14)-\mathrm{H}(12)$ & $0.90(2)$ \\
\hline $\mathrm{C}(15)-\mathrm{O}(5)$ & $1.420(2)$ \\
\hline$C(15)-C(17)$ & $1.551(3)$ \\
\hline $\mathrm{C}(15)-\mathrm{H}(13)$ & $0.94(2)$ \\
\hline $\mathrm{C}(16)-\mathrm{O}(5)$ & $1.428(2)$ \\
\hline$C(16)-C(20)$ & $1.541(3)$ \\
\hline $\mathrm{C}(16)-\mathrm{H}(14)$ & $0.94(2)$ \\
\hline $\mathrm{C}(17)-\mathrm{C}(18)$ & $1.507(3)$ \\
\hline $\mathrm{C}(17)-\mathrm{H}(15)$ & $0.94(3)$ \\
\hline $\mathrm{C}(17)-\mathrm{H}(16)$ & $0.93(3)$ \\
\hline $\mathrm{C}(18)-\mathrm{O}(4)$ & $1.219(3)$ \\
\hline $\mathrm{C}(18)-\mathrm{C}(19)$ & $1.512(3)$ \\
\hline$C(19)-C(20)$ & $1.530(3)$ \\
\hline $\mathrm{C}(19)-\mathrm{H}(17)$ & $0.92(3)$ \\
\hline $\mathrm{C}(19)-\mathrm{H}(18)$ & $0.91(3)$ \\
\hline $\mathrm{C}(20)-\mathrm{H}(19)$ & $0.96(3)$ \\
\hline $\mathrm{C}(20)-\mathrm{H}(20)$ & $0.95(3)$ \\
\hline $\mathrm{C}(21)-\mathrm{O}(3)$ & $1.435(3)$ \\
\hline $\mathrm{C}(21)-\mathrm{H}(21)$ & $0.99(3)$ \\
\hline $\mathrm{C}(21)-\mathrm{H}(22)$ & $0.96(3)$ \\
\hline $\mathrm{C}(21)-\mathrm{H}(23)$ & $0.94(3)$ \\
\hline $\mathrm{C}(1 \mathrm{~S})-\mathrm{Cl}(1 \mathrm{~S})$ & $1.750(2)$ \\
\hline $\mathrm{C}(1 \mathrm{~S})-\mathrm{Cl}(2 \mathrm{~S})$ & $1.760(3)$ \\
\hline $\mathrm{C}(1 \mathrm{~S})-\mathrm{Cl}(3 \mathrm{~S})$ & $1.770(3)$ \\
\hline
\end{tabular}




$\begin{array}{ll}\mathrm{C}(1 \mathrm{~S})-\mathrm{H}(1 \mathrm{~S}) & 0.88(3) \\ \mathrm{Mo}(1)-\mathrm{N}(2) & 2.2076(17) \\ \mathrm{Mo}(1)-\mathrm{N}(6) & 2.2377(17) \\ \mathrm{Mo}(1)-\mathrm{N}(4) & 2.2862(17) \\ \mathrm{N}(1)-\mathrm{N}(2) & 1.368(2) \\ \mathrm{N}(3)-\mathrm{N}(4) & 1.363(2) \\ \mathrm{N}(5)-\mathrm{N}(6) & 1.367(2)\end{array}$

$$
\begin{array}{ll}
\mathrm{N}(5)-\mathrm{B}(1)-\mathrm{N}(1) & 107.32(17) \\
\mathrm{N}(5)-\mathrm{B}(1)-\mathrm{N}(3) & 110.20(17) \\
\mathrm{N}(1)-\mathrm{B}(1)-\mathrm{N}(3) & 107.76(17) \\
\mathrm{N}(5)-\mathrm{B}(1)-\mathrm{H}(10) & 110.9(13) \\
\mathrm{N}(1)-\mathrm{B}(1)-\mathrm{H}(10) & 112.2(13) \\
\mathrm{N}(3)-\mathrm{B}(1)-\mathrm{H}(10) & 108.3(13) \\
\mathrm{N}(1)-\mathrm{C}(1)-\mathrm{C}(2) & 109.0(2) \\
\mathrm{N}(1)-\mathrm{C}(1)-\mathrm{H}(1) & 119.7(17) \\
\mathrm{C}(2)-\mathrm{C}(1)-\mathrm{H}(1) & 131.2(17) \\
\mathrm{C}(1)-\mathrm{C}(2)-\mathrm{C}(3) & 104.6(2) \\
\mathrm{C}(1)-\mathrm{C}(2)-\mathrm{H}(2) & 128.9(19) \\
\mathrm{C}(3)-\mathrm{C}(2)-\mathrm{H}(2) & 126.5(19) \\
\mathrm{N}(2)-\mathrm{C}(3)-\mathrm{C}(2) & 111.0(2) \\
\mathrm{N}(2)-\mathrm{C}(3)-\mathrm{H}(3) & 118.0(16) \\
\mathrm{C}(2)-\mathrm{C}(3)-\mathrm{H}(3) & 131.0(16) \\
\mathrm{N}(3)-\mathrm{C}(4)-\mathrm{C}(5) & 108.81(19) \\
\mathrm{N}(3)-\mathrm{C}(4)-\mathrm{H}(4) & 118.9(17) \\
\mathrm{C}(5)-\mathrm{C}(4)-\mathrm{H}(4) & 132.3(17) \\
\mathrm{C}(4)-\mathrm{C}(5)-\mathrm{C}(6) & 104.5(2) \\
& \\
& \\
& \\
&
\end{array}
$$


$\mathrm{C}(4)-\mathrm{C}(5)-\mathrm{H}(5) \quad 126.7(19)$

$\mathrm{C}(6)-\mathrm{C}(5)-\mathrm{H}(5) \quad 128.7(19)$

$\mathrm{N}(4)-\mathrm{C}(6)-\mathrm{C}(5) \quad 111.0(2)$

$\mathrm{N}(4)-\mathrm{C}(6)-\mathrm{H}(6) \quad 118.7(16)$

$\mathrm{C}(5)-\mathrm{C}(6)-\mathrm{H}(6) \quad 130.3(16)$

$\mathrm{N}(5)-\mathrm{C}(7)-\mathrm{C}(8) \quad 108.6(2)$

$\mathrm{N}(5)-\mathrm{C}(7)-\mathrm{H}(7) \quad 120.2(18)$

$\mathrm{C}(8)-\mathrm{C}(7)-\mathrm{H}(7) \quad 131.3(18)$

$\mathrm{C}(7)-\mathrm{C}(8)-\mathrm{C}(9) \quad 105.3(2)$

$\mathrm{C}(7)-\mathrm{C}(8)-\mathrm{H}(8) \quad 129.0(19)$

$\mathrm{C}(9)-\mathrm{C}(8)-\mathrm{H}(8) \quad 125.8(19)$

$\mathrm{N}(6)-\mathrm{C}(9)-\mathrm{C}(8) \quad 110.6(2)$

$\mathrm{N}(6)-\mathrm{C}(9)-\mathrm{H}(9) \quad 115.8(15)$

$\mathrm{C}(8)-\mathrm{C}(9)-\mathrm{H}(9) \quad 133.6(16)$

$\mathrm{O}(1)-\mathrm{C}(10)-\mathrm{Mo}(1)$ 176.64(17)

$\mathrm{O}(2)-\mathrm{C}(11)-\mathrm{Mo}(1) \quad 177.98(18)$

$\mathrm{O}(3)-\mathrm{C}(12)-\mathrm{C}(13) \quad 123.97(17)$

$\mathrm{O}(3)-\mathrm{C}(12)-\mathrm{C}(16)$ 108.95(16)

C(13)-C(12)-C(16)120.87(17)

O(3)-C(12)-Mo(1) 119.92(13)

C(13)-C(12)-Mo(1) 60.92(11)

C(16)-C(12)-Mo(1)114.33(13)

C(12)-C(13)-C(14)114.08(17)

C(12)-C(13)-Mo(1) 86.15(12)

C(14)-C(13)-Mo(1) 73.66(11)

C(12)-C(13)-H(11)121.7(14)

C(14)-C(13)-H(11)124.2(14) 
Mo(1)-C(13)-H(11)110.3(14)

$\mathrm{C}(13)-\mathrm{C}(14)-\mathrm{C}(15) 116.32(17)$

C(13)-C(14)-Mo(1) 69.30(11)

C(15)-C(14)-Mo(1)120.65(13)

$\mathrm{C}(13)-\mathrm{C}(14)-\mathrm{H}(12) 115.5(15)$

$\mathrm{C}(15)-\mathrm{C}(14)-\mathrm{H}(12) 112.3(15)$

Mo(1)-C(14)-H(12)116.3(15)

$\mathrm{O}(5)-\mathrm{C}(15)-\mathrm{C}(14) 112.43(16)$

$\mathrm{O}(5)-\mathrm{C}(15)-\mathrm{C}(17) 112.09(18)$

$\mathrm{C}(14)-\mathrm{C}(15)-\mathrm{C}(17) 111.02(17)$

$\mathrm{O}(5)-\mathrm{C}(15)-\mathrm{H}(13)$ 102.2(15)

$\mathrm{C}(14)-\mathrm{C}(15)-\mathrm{H}(13) 112.4(15)$

$\mathrm{C}(17)-\mathrm{C}(15)-\mathrm{H}(13) 106.2(15)$

$\mathrm{O}(5)-\mathrm{C}(16)-\mathrm{C}(12) 112.39(16)$

$\mathrm{O}(5)-\mathrm{C}(16)-\mathrm{C}(20)$ 111.34(17)

$\mathrm{C}(12)-\mathrm{C}(16)-\mathrm{C}(20) 109.74(17)$

$\mathrm{O}(5)-\mathrm{C}(16)-\mathrm{H}(14)$ 103.8(14)

$\mathrm{C}(12)-\mathrm{C}(16)-\mathrm{H}(14) 110.4(14)$

C(20)-C(16)-H(14)109.0(14)

C(18)-C(17)-C(15)114.76(17)

C(18)-C(17)-H(15)107.6(16)

C(15)-C(17)-H(15)108.7(16)

C(18)-C(17)-H(16)109.3(17)

C(15)-C(17)-H(16)108.8(16)

$\mathrm{H}(15)-\mathrm{C}(17)-\mathrm{H}(16) 107(2)$

$\mathrm{O}(4)-\mathrm{C}(18)-\mathrm{C}(17) 119.9(2)$

$\mathrm{O}(4)-\mathrm{C}(18)-\mathrm{C}(19) 119.9(2)$ 
C(17)-C(18)-C(19)120.2(2)

C(18)-C(19)-C(20)118.88(19)

C(18)-C(19)-H(17)106.4(16)

C(20)-C(19)-H(17)111.6(16)

C(18)-C(19)-H(18)104(2)

$\mathrm{C}(20)-\mathrm{C}(19)-\mathrm{H}(18) 111.0(19)$

H(17)-C(19)-H(18)104(2)

C(19)-C(20)-C(16)116.34(18)

C(19)-C(20)-H(19)111.7(15)

$\mathrm{C}(16)-\mathrm{C}(20)-\mathrm{H}(19) 105.9(15)$

C(19)-C(20)-H(20)106.8(16)

$\mathrm{C}(16)-\mathrm{C}(20)-\mathrm{H}(20) 105.4(15)$

H(19)-C(20)-H(20)111(2)

$\mathrm{O}(3)-\mathrm{C}(21)-\mathrm{H}(21)$ 110.3(16)

$\mathrm{O}(3)-\mathrm{C}(21)-\mathrm{H}(22) 111.9(15)$

$\mathrm{H}(21)-\mathrm{C}(21)-\mathrm{H}(22) 110(2)$

$\mathrm{O}(3)-\mathrm{C}(21)-\mathrm{H}(23)$ 105.6(17)

$\mathrm{H}(21)-\mathrm{C}(21)-\mathrm{H}(23) 110(2)$

$\mathrm{H}(22)-\mathrm{C}(21)-\mathrm{H}(23) 109(2)$

Cl(1S)-C(1S)-Cl(2S)110.44(14)

Cl(1S)-C(1S)-Cl(3S)108.71(13)

Cl(2S)-C(1S)-Cl(3S)110.69(14)

Cl(1S)-C(1S)-H(1S)109.1(19)

Cl(2S)-C(1S)-H(1S)108.7(19)

Cl(3S)-C(1S)-H(1S)109.1(19)

C(10)-Mo(1)-C(11) 83.61(8)

$\mathrm{C}(10)-\mathrm{Mo}(1)-\mathrm{N}(2) \quad 82.19(7)$ 
C(11)-Mo(1)-N(2) 95.47(7)

C(10)-Mo(1)-C(13)102.65(8)

C(11)-Mo(1)-C(13)103.86(8)

$\mathrm{N}(2)-\mathrm{Mo}(1)-\mathrm{C}(13) 160.45(7)$

C(10)-Mo(1)-N(6) 158.53(7)

C(11)-Mo(1)-N(6) 88.82(7)

$\mathrm{N}(2)-\mathrm{Mo}(1)-\mathrm{N}(6) \quad 78.57(6)$

C(13)-Mo(1)-N(6) 98.66(7)

$\mathrm{C}(10)-\mathrm{Mo}(1)-\mathrm{N}(4) 101.57(7)$

$\mathrm{C}(11)-\mathrm{Mo}(1)-\mathrm{N}(4) 172.20(7)$

$\mathrm{N}(2)-\mathrm{Mo}(1)-\mathrm{N}(4) \quad 79.61(6)$

C(13)-Mo(1)-N(4) 80.85(7)

$\mathrm{N}(6)-\mathrm{Mo}(1)-\mathrm{N}(4) \quad 84.29(6)$

$\mathrm{C}(10)-\mathrm{Mo}(1)-\mathrm{C}(14) 65.75(8)$

$\mathrm{C}(11)-\mathrm{Mo}(1)-\mathrm{C}(14) 101.93(8)$

N(2)-Mo(1)-C(14)141.07(7)

C(13)-Mo(1)-C(14) 37.05(7)

N(6)-Mo(1)-C(14)135.66(7)

$\mathrm{N}(4)-\mathrm{Mo}(1)-\mathrm{C}(14) \quad 85.59(7)$

C(10)-Mo(1)-C(12)112.16(7)

$\mathrm{C}(11)-\mathrm{Mo}(1)-\mathrm{C}(12) 73.90(7)$

N(2)-Mo(1)-C(12)160.40(6)

C(13)-Mo(1)-C(12) 32.92(7)

$\mathrm{N}(6)-\mathrm{Mo}(1)-\mathrm{C}(12) \quad 84.69(6)$

$\mathrm{N}(4)-\mathrm{Mo}(1)-\mathrm{C}(12) 108.97(6)$

C(14)-Mo(1)-C(12) 58.40(7)

$\mathrm{C}(1)-\mathrm{N}(1)-\mathrm{N}(2) \quad 109.40(17)$ 


$$
\begin{array}{ll}
\mathrm{C}(1)-\mathrm{N}(1)-\mathrm{B}(1) & 130.13(19) \\
\mathrm{N}(2)-\mathrm{N}(1)-\mathrm{B}(1) & 120.46(16) \\
\mathrm{C}(3)-\mathrm{N}(2)-\mathrm{N}(1) & 105.97(17) \\
\mathrm{C}(3)-\mathrm{N}(2)-\mathrm{Mo}(1) & 131.88(15) \\
\mathrm{N}(1)-\mathrm{N}(2)-\mathrm{Mo}(1) & 122.07(12) \\
\mathrm{C}(4)-\mathrm{N}(3)-\mathrm{N}(4) & 109.70(17) \\
\mathrm{C}(4)-\mathrm{N}(3)-\mathrm{B}(1) & 128.95(18) \\
\mathrm{N}(4)-\mathrm{N}(3)-\mathrm{B}(1) & 121.14(16) \\
\mathrm{C}(6)-\mathrm{N}(4)-\mathrm{N}(3) & 105.95(17) \\
\mathrm{C}(6)-\mathrm{N}(4)-\mathrm{Mo}(1) & 134.25(15) \\
\mathrm{N}(3)-\mathrm{N}(4)-\mathrm{Mo}(1) & 119.63(12) \\
\mathrm{C}(7)-\mathrm{N}(5)-\mathrm{N}(6) & 109.22(18) \\
\mathrm{C}(7)-\mathrm{N}(5)-\mathrm{B}(1) & 129.23(19) \\
\mathrm{N}(6)-\mathrm{N}(5)-\mathrm{B}(1) & 120.09(16) \\
\mathrm{C}(9)-\mathrm{N}(6)-\mathrm{N}(5) & 106.39(17) \\
\mathrm{C}(9)-\mathrm{N}(6)-\mathrm{Mo}(1) & 131.07(15) \\
\mathrm{N}(5)-\mathrm{N}(6)-\mathrm{Mo}(1) & 121.86(13) \\
\mathrm{C}(12)-\mathrm{O}(3)-\mathrm{C}(21) & 117.46(16) \\
\mathrm{C}(15)-\mathrm{O}(5)-\mathrm{C}(16) & 115.07(15)
\end{array}
$$

Table S5. Anisotropic displacement parameters $\left(\AA^{2} \times 10^{3}\right)$ for $( \pm)-13$. The anisotropic displacement factor exponent takes the form: $-2 \pi^{2}\left[\mathrm{~h}^{2} \mathrm{a}^{*} \mathrm{U}^{11}+\ldots+2 \mathrm{hk} \mathrm{a}^{*} \mathrm{~b}^{*} \mathrm{U}^{12}\right]$

\begin{tabular}{lllllll}
\hline & $\mathrm{U}^{11}$ & $\mathrm{U}^{22}$ & $\mathrm{U}^{33}$ & $\mathrm{U}^{23}$ & $\mathrm{U}^{13}$ & $\mathrm{U}^{12}$ \\
\hline $\mathrm{B}(1)$ & $25(1)$ & $24(1)$ & $28(1)$ & $-1(1)$ & $-6(1)$ & $-6(1)$ \\
$\mathrm{C}(1)$ & $28(1)$ & $36(1)$ & $29(1)$ & $10(1)$ & $-10(1)$ & $-16(1)$ \\
$\mathrm{C}(2)$ & $39(1)$ & $42(1)$ & $26(1)$ & $5(1)$ & $-12(1)$ & $-24(1)$
\end{tabular}




\begin{tabular}{|c|c|c|c|c|c|c|}
\hline$C(3)$ & $34(1)$ & $31(1)$ & $20(1)$ & $0(1)$ & $-4(1)$ & $-17(1)$ \\
\hline$C(4)$ & $23(1)$ & $31(1)$ & $31(1)$ & $-9(1)$ & $-1(1)$ & $-8(1)$ \\
\hline$C(5)$ & $28(1)$ & $41(1)$ & $24(1)$ & $-6(1)$ & $4(1)$ & $-14(1)$ \\
\hline$C(6)$ & $25(1)$ & $32(1)$ & $23(1)$ & $1(1)$ & $-1(1)$ & $-12(1)$ \\
\hline$C(7)$ & $44(1)$ & $19(1)$ & $30(1)$ & $1(1)$ & $-11(1)$ & $-11(1)$ \\
\hline$C(8)$ & $52(1)$ & $28(1)$ & $30(1)$ & $6(1)$ & $-12(1)$ & $-25(1)$ \\
\hline$C(9)$ & $34(1)$ & $28(1)$ & $26(1)$ & $3(1)$ & $-7(1)$ & $-18(1)$ \\
\hline$C(10)$ & $27(1)$ & $24(1)$ & $18(1)$ & $-1(1)$ & $-1(1)$ & $-11(1)$ \\
\hline$C(11)$ & $28(1)$ & $20(1)$ & $23(1)$ & $2(1)$ & $-5(1)$ & $-12(1)$ \\
\hline$C(12)$ & $22(1)$ & $21(1)$ & $24(1)$ & $-1(1)$ & $-5(1)$ & $-8(1)$ \\
\hline$C(13)$ & $21(1)$ & $19(1)$ & $20(1)$ & $0(1)$ & $-4(1)$ & $-6(1)$ \\
\hline$C(14)$ & $24(1)$ & $22(1)$ & $20(1)$ & $1(1)$ & $-2(1)$ & $-9(1)$ \\
\hline$C(15)$ & $28(1)$ & $20(1)$ & $26(1)$ & $-1(1)$ & $-4(1)$ & $-8(1)$ \\
\hline$C(16)$ & $20(1)$ & $23(1)$ & $26(1)$ & $-1(1)$ & $-1(1)$ & $-7(1)$ \\
\hline$C(17)$ & $31(1)$ & $21(1)$ & $31(1)$ & $2(1)$ & $-7(1)$ & $-6(1)$ \\
\hline$C(18)$ & $29(1)$ & $25(1)$ & $32(1)$ & $2(1)$ & $-13(1)$ & $-1(1)$ \\
\hline$C(19)$ & $29(1)$ & $29(1)$ & $36(1)$ & $-2(1)$ & $-13(1)$ & $-6(1)$ \\
\hline$C(20)$ & $21(1)$ & $30(1)$ & $40(1)$ & $-1(1)$ & $-6(1)$ & $-8(1)$ \\
\hline $\mathrm{C}(21)$ & $34(1)$ & $24(1)$ & $37(1)$ & $-5(1)$ & $-8(1)$ & $-13(1)$ \\
\hline $\mathrm{C}(1 \mathrm{~S})$ & $36(1)$ & $33(1)$ & $26(1)$ & $2(1)$ & $-1(1)$ & $-8(1)$ \\
\hline $\mathrm{Cl}(1 \mathrm{~S})$ & $44(1)$ & $40(1)$ & $32(1)$ & $12(1)$ & $-5(1)$ & $-17(1)$ \\
\hline $\mathrm{Cl}(2 \mathrm{~S})$ & $51(1)$ & $63(1)$ & $34(1)$ & $6(1)$ & $-17(1)$ & $-13(1)$ \\
\hline $\mathrm{Cl}(3 \mathrm{~S})$ & $40(1)$ & $53(1)$ & $71(1)$ & $15(1)$ & $-1(1)$ & $-25(1)$ \\
\hline $\operatorname{Mo}(1)$ & $19(1)$ & $17(1)$ & $18(1)$ & $-1(1)$ & $-1(1)$ & $-8(1)$ \\
\hline $\mathrm{N}(1)$ & $24(1)$ & $24(1)$ & $24(1)$ & $1(1)$ & $-5(1)$ & $-9(1)$ \\
\hline $\mathrm{N}(2)$ & $24(1)$ & $22(1)$ & $22(1)$ & $-1(1)$ & $-3(1)$ & $-10(1)$ \\
\hline $\mathrm{N}(3)$ & $21(1)$ & $25(1)$ & $25(1)$ & $-3(1)$ & $-2(1)$ & $-7(1)$ \\
\hline
\end{tabular}




$\begin{array}{lllllll}\mathrm{N}(4) & 22(1) & 25(1) & 23(1) & -1(1) & -4(1) & -9(1) \\ \mathrm{N}(5) & 29(1) & 19(1) & 28(1) & 1(1) & -7(1) & -8(1) \\ \mathrm{N}(6) & 25(1) & 21(1) & 27(1) & 2(1) & -6(1) & -10(1) \\ \mathrm{O}(1) & 46(1) & 26(1) & 27(1) & 1(1) & -5(1) & -20(1) \\ \mathrm{O}(2) & 34(1) & 34(1) & 25(1) & -2(1) & 4(1) & -13(1) \\ \mathrm{O}(3) & 24(1) & 23(1) & 34(1) & -2(1) & -3(1) & -12(1) \\ \mathrm{O}(4) & 49(1) & 42(1) & 29(1) & -2(1) & -6(1) & -14(1) \\ \mathrm{O}(5) 25(1) & 21(1) & 25(1) & -3(1) & 0(1) & -7(1) & \end{array}$

Table S6. Hydrogen coordinates ( x 10 $0^{4}$ and isotropic displacement parameters $\left(\AA^{2} \times 10^{3}\right)$ for ( $( \pm)-13$.

$\begin{array}{llll}\text { x } & \mathrm{y} & \mathrm{U} & \mathrm{U}(\mathrm{eq})\end{array}$

$\begin{array}{lrrrr}\mathrm{H}(1) & 15510(30) & 5470(30) & 4237(16) & 27(6) \\ \mathrm{H}(2) & 14420(40) & 7630(30) & 5210(20) & 42(8) \\ \mathrm{H}(3) & 11650(30) & 8980(30) & 4778(16) & 23(6) \\ \mathrm{H}(4) & 15460(30) & 4680(30) & 1384(18) & 34(7) \\ \mathrm{H}(5) & 14550(40) & 6420(30) & 260(20) & 41(8) \\ \mathrm{H}(6) & 11910(30) & 8250(30) & 1101(16) & 23(6) \\ \mathrm{H}(7) & 12830(30) & 2920(30) & 3654(18) & 35(7) \\ \mathrm{H}(8) & 9830(30) & 3370(30) & 4260(18) & 36(7) \\ \mathrm{H}(9) & 8250(30) & 5870(30) & 4038(16) & 25(6) \\ \mathrm{H}(10) & 14640(30) & 4310(30) & 2999(16) & 26(6) \\ \mathrm{H}(11) & 9250(30) & 7600(20) & 1649(16) & 24(6)\end{array}$




$\begin{array}{lllll}\mathrm{H}(12) & 9480(30) & 9750(20) & 1805(16) & 23(6) \\ \mathrm{H}(13) & 7370(30) & 11530(30) & 2779(16) & 23(6) \\ \mathrm{H}(14) & 5320(30) & 9190(20) & 3700(16) & 22(6) \\ \mathrm{H}(15) & 6860(30) & 11840(30) & 1404(17) & 32(7) \\ \mathrm{H}(16) & 5200(30) & 12050(30) & 1981(17) & 31(7) \\ \mathrm{H}(17) & 5650(30) & 8640(30) & 1336(17) & 28(6) \\ \mathrm{H}(18) & 4180(40) & 9830(30) & 1250(20) & 49(8) \\ \mathrm{H}(19) & 3580(30) & 10500(30) & 2743(16) & 25(6) \\ \mathrm{H}(20) & 4000(30) & 8920(30) & 2661(17) & 29(6) \\ \mathrm{H}(21) & 7710(30) & 6170(30) & 1524(19) & 34(7) \\ \mathrm{H}(22) & 9130(30) & 5590(30) & 2116(16) & 26(6) \\ \mathrm{H}(23) & 7610(30) & 5160(30) & 2271(18) & 38(7) \\ \mathrm{H}(1 \mathrm{~S}) & 1950(40) & 1810(30) & 466(19) & 41(8)\end{array}$

Table S7. Torsion angles $\left[^{\circ}\right]$ for $( \pm)-\mathbf{1 3}$.

$\begin{array}{lc}\mathrm{N}(1)-\mathrm{C}(1)-\mathrm{C}(2)-\mathrm{C}(3) & -0.2(2) \\ \mathrm{C}(1)-\mathrm{C}(2)-\mathrm{C}(3)-\mathrm{N}(2) & 0.3(3) \\ \mathrm{N}(3)-\mathrm{C}(4)-\mathrm{C}(5)-\mathrm{C}(6) & 0.5(3) \\ \mathrm{C}(4)-\mathrm{C}(5)-\mathrm{C}(6)-\mathrm{N}(4) & -0.6(3) \\ \mathrm{N}(5)-\mathrm{C}(7)-\mathrm{C}(8)-\mathrm{C}(9) & -1.1(2) \\ \mathrm{C}(7)-\mathrm{C}(8)-\mathrm{C}(9)-\mathrm{N}(6) & 1.2(3) \\ \mathrm{O}(3)-\mathrm{C}(12)-\mathrm{C}(13)-\mathrm{C}(14) & -178.47(17) \\ \mathrm{C}(16)-\mathrm{C}(12)-\mathrm{C}(13)-\mathrm{C}(14) & 32.3(3) \\ \mathrm{Mo}(1)-\mathrm{C}(12)-\mathrm{C}(13)-\mathrm{C}(14) & -70.19(15) \\ \mathrm{O}(3)-\mathrm{C}(12)-\mathrm{C}(13)-\mathrm{Mo}(1) & -108.28(18) \\ \mathrm{C}(16)-\mathrm{C}(12)-\mathrm{C}(13)-\mathrm{Mo}(1) & 102.53(17)\end{array}$




\begin{tabular}{|c|c|}
\hline$C(12)-C(13)-C(14)-C(15)$ & $-36.9(2)$ \\
\hline $\mathrm{Mo}(1)-\mathrm{C}(13)-\mathrm{C}(14)-\mathrm{C}(15)$ & $-114.90(16)$ \\
\hline $\mathrm{C}(12)-\mathrm{C}(13)-\mathrm{C}(14)-\mathrm{Mo}(1)$ & $78.02(15)$ \\
\hline $\mathrm{C}(13)-\mathrm{C}(14)-\mathrm{C}(15)-\mathrm{O}(5)$ & $46.7(2)$ \\
\hline $\mathrm{Mo}(1)-\mathrm{C}(14)-\mathrm{C}(15)-\mathrm{O}(5)$ & $-33.8(2)$ \\
\hline$C(13)-C(14)-C(15)-C(17)$ & $-79.8(2)$ \\
\hline $\mathrm{Mo}(1)-\mathrm{C}(14)-\mathrm{C}(15)-\mathrm{C}(17)$ & $-160.28(14)$ \\
\hline $\mathrm{O}(3)-\mathrm{C}(12)-\mathrm{C}(16)-\mathrm{O}(5)$ & $170.58(16)$ \\
\hline $\mathrm{C}(13)-\mathrm{C}(12)-\mathrm{C}(16)-\mathrm{O}(5)$ & $-36.1(3)$ \\
\hline $\mathrm{Mo}(1)-\mathrm{C}(12)-\mathrm{C}(16)-\mathrm{O}(5)$ & $33.3(2)$ \\
\hline $\mathrm{O}(3)-\mathrm{C}(12)-\mathrm{C}(16)-\mathrm{C}(20)$ & $-65.0(2)$ \\
\hline$C(13)-C(12)-C(16)-C(20)$ & $88.4(2)$ \\
\hline $\mathrm{Mo}(1)-\mathrm{C}(12)-\mathrm{C}(16)-\mathrm{C}(20)$ & $157.81(14)$ \\
\hline $\mathrm{O}(5)-\mathrm{C}(15)-\mathrm{C}(17)-\mathrm{C}(18)$ & $-79.6(2)$ \\
\hline$C(14)-C(15)-C(17)-C(18)$ & $47.0(3)$ \\
\hline $\mathrm{C}(15)-\mathrm{C}(17)-\mathrm{C}(18)-\mathrm{O}(4)$ & $-116.0(2)$ \\
\hline$C(15)-C(17)-C(18)-C(19)$ & $64.9(3)$ \\
\hline $\mathrm{O}(4)-\mathrm{C}(18)-\mathrm{C}(19)-\mathrm{C}(20)$ & $169.8(2)$ \\
\hline $\mathrm{C}(17)-\mathrm{C}(18)-\mathrm{C}(19)-\mathrm{C}(20)$ & $-11.1(3)$ \\
\hline $\mathrm{C}(18)-\mathrm{C}(19)-\mathrm{C}(20)-\mathrm{C}(16)$ & $-51.1(3)$ \\
\hline $\mathrm{O}(5)-\mathrm{C}(16)-\mathrm{C}(20)-\mathrm{C}(19)$ & $82.0(2)$ \\
\hline$C(12)-C(16)-C(20)-C(19)$ & $-43.1(3)$ \\
\hline $\mathrm{O}(1)-\mathrm{C}(10)-\mathrm{Mo}(1)-\mathrm{C}(11)$ & $31(3)$ \\
\hline $\mathrm{O}(1)-\mathrm{C}(10)-\mathrm{Mo}(1)-\mathrm{N}(2)$ & $-65(3)$ \\
\hline $\mathrm{O}(1)-\mathrm{C}(10)-\mathrm{Mo}(1)-\mathrm{C}(13)$ & $134(3)$ \\
\hline $\mathrm{O}(1)-\mathrm{C}(10)-\mathrm{Mo}(1)-\mathrm{N}(6)$ & $-39(3)$ \\
\hline $\mathrm{O}(1)-\mathrm{C}(10)-\mathrm{Mo}(1)-\mathrm{N}(4)$ & $-143(3)$ \\
\hline
\end{tabular}




$$
\begin{aligned}
& \mathrm{O}(1)-\mathrm{C}(10)-\mathrm{Mo}(1)-\mathrm{C}(14) \quad 138(3) \\
& \mathrm{O}(1)-\mathrm{C}(10)-\mathrm{Mo}(1)-\mathrm{C}(12) \quad \text { 101(3) } \\
& \mathrm{O}(2)-\mathrm{C}(11)-\mathrm{Mo}(1)-\mathrm{C}(10) \quad-122(5) \\
& \mathrm{O}(2)-\mathrm{C}(11)-\mathrm{Mo}(1)-\mathrm{N}(2) \quad-40(5) \\
& \mathrm{O}(2)-\mathrm{C}(11)-\mathrm{Mo}(1)-\mathrm{C}(13) \quad 137(5) \\
& \mathrm{O}(2)-\mathrm{C}(11)-\mathrm{Mo}(1)-\mathrm{N}(6) \\
& \mathrm{O}(2)-\mathrm{C}(11)-\mathrm{Mo}(1)-\mathrm{N}(4) \quad 10(5) \\
& \mathrm{O}(2)-\mathrm{C}(11)-\mathrm{Mo}(1)-\mathrm{C}(14) \quad 175(5) \\
& \mathrm{O}(2)-\mathrm{C}(11)-\mathrm{Mo}(1)-\mathrm{C}(12) \quad 123(5) \\
& \mathrm{C}(12)-\mathrm{C}(13)-\mathrm{Mo}(1)-\mathrm{C}(10) \quad-111.40(12) \\
& \text { C(14)-C(13)-Mo(1)-C(10) 5.08(13) } \\
& \mathrm{C}(12)-\mathrm{C}(13)-\mathrm{Mo}(1)-\mathrm{C}(11) \quad-24.96(13) \\
& \text { C(14)-C(13)-Mo(1)-C(11) 91.53(12) } \\
& \mathrm{C}(12)-\mathrm{C}(13)-\mathrm{Mo}(1)-\mathrm{N}(2) \quad 146.16(18) \\
& \mathrm{C}(14)-\mathrm{C}(13)-\mathrm{Mo}(1)-\mathrm{N}(2) \quad-97.4(2) \\
& \text { C(12)-C(13)-Mo(1)-N(6) 65.96(12) } \\
& \text { C(14)-C(13)-Mo(1)-N(6) - } \quad-177.56(11) \\
& \text { C(12)-C(13)-Mo(1)-N(4) 148.70(12) } \\
& \text { C(14)-C(13)-Mo(1)-N(4) -94.82(12) } \\
& \text { C(12)-C(13)-Mo(1)-C(14) -116.48(16) } \\
& \text { C(14)-C(13)-Mo(1)-C(12) 116.48(16) } \\
& \text { C(13)-C(14)-Mo(1)-C(10) - -174.56(14) } \\
& \text { C(15)-C(14)-Mo(1)-C(10) -65.47(16) } \\
& \text { C(13)-C(14)-Mo(1)-C(11) -97.26(12) } \\
& \mathrm{C}(15)-\mathrm{C}(14)-\mathrm{Mo}(1)-\mathrm{C}(11) \quad 11.83(17) \\
& \text { C(13)-C(14)-Mo(1)-N(2) 148.11(12) } \\
& \mathrm{C}(15)-\mathrm{C}(14)-\mathrm{Mo}(1)-\mathrm{N}(2) \quad-102.80(17)
\end{aligned}
$$




$$
\begin{aligned}
& \text { C(15)-C(14)-Mo(1)-C(13) 109.1(2) } \\
& \text { C(13)-C(14)-Mo(1)-N(6) 3.46(16) } \\
& \text { C(15)-C(14)-Mo(1)-N(6) 112.54(16) } \\
& \text { C(13)-C(14)-Mo(1)-N(4) 80.65(11) } \\
& \text { C(15)-C(14)-Mo(1)-N(4) -170.26(16) } \\
& \mathrm{C}(13)-\mathrm{C}(14)-\mathrm{Mo}(1)-\mathrm{C}(12) \quad-34.83(11) \\
& \text { C(15)-C(14)-Mo(1)-C(12) 74.26(16) } \\
& \mathrm{O}(3)-\mathrm{C}(12)-\mathrm{Mo}(1)-\mathrm{C}(10) \quad-166.51(14) \\
& \text { C(13)-C(12)-Mo(1)-C(10) 78.80(13) } \\
& \text { C(16)-C(12)-Mo(1)-C(10) -34.33(16) } \\
& \mathrm{O}(3)-\mathrm{C}(12)-\mathrm{Mo}(1)-\mathrm{C}(11) \quad-90.56(15) \\
& \text { C(13)-C(12)-Mo(1)-C(11) 154.76(13) } \\
& \text { C(16)-C(12)-Mo(1)-C(11) 41.63(14) } \\
& \mathrm{O}(3)-\mathrm{C}(12)-\mathrm{Mo}(1)-\mathrm{N}(2) \quad-31.6(3) \\
& \text { C(13)-C(12)-Mo(1)-N(2) - -146.24(18) } \\
& \mathrm{C}(16)-\mathrm{C}(12)-\mathrm{Mo}(1)-\mathrm{N}(2) \quad 100.6(2) \\
& \mathrm{O}(3)-\mathrm{C}(12)-\mathrm{Mo}(1)-\mathrm{C}(13) \quad 114.7(2) \\
& \text { C(16)-C(12)-Mo(1)-C(13) - -113.13(19) } \\
& \mathrm{O}(3)-\mathrm{C}(12)-\mathrm{Mo}(1)-\mathrm{N}(6) \quad-0.26(15) \\
& \text { C(13)-C(12)-Mo(1)-N(6) -114.94(12) } \\
& \text { C(16)-C(12)-Mo(1)-N(6) 131.93(14) } \\
& \mathrm{O}(3)-\mathrm{C}(12)-\mathrm{Mo}(1)-\mathrm{N}(4) \quad 81.83(15) \\
& \text { C(13)-C(12)-Mo(1)-N(4) -32.85(13) } \\
& \text { C(16)-C(12)-Mo(1)-N(4) - -145.98(13) } \\
& \mathrm{O}(3)-\mathrm{C}(12)-\mathrm{Mo}(1)-\mathrm{C}(14) \quad 153.96(17) \\
& \text { C(13)-C(12)-Mo(1)-C(14) 39.28(12) } \\
& \mathrm{C}(16)-\mathrm{C}(12)-\mathrm{Mo}(1)-\mathrm{C}(14) \quad-73.85(14)
\end{aligned}
$$




$$
\begin{aligned}
& \mathrm{C}(2)-\mathrm{C}(1)-\mathrm{N}(1)-\mathrm{N}(2) \quad-0.1(2) \\
& \mathrm{C}(2)-\mathrm{C}(1)-\mathrm{N}(1)-\mathrm{B}(1) \quad-179.1(2) \\
& \mathrm{N}(5)-\mathrm{B}(1)-\mathrm{N}(1)-\mathrm{C}(1) \\
& \mathrm{N}(3)-\mathrm{B}(1)-\mathrm{N}(1)-\mathrm{C}(1) \quad-119.5(2) \\
& \mathrm{N}(5)-\mathrm{B}(1)-\mathrm{N}(1)-\mathrm{N}(2) \quad-57.1(2) \\
& \mathrm{N}(3)-\mathrm{B}(1)-\mathrm{N}(1)-\mathrm{N}(2) \\
& \mathrm{C}(2)-\mathrm{C}(3)-\mathrm{N}(2)-\mathrm{N}(1) \quad-0.3(2) \\
& \mathrm{C}(2)-\mathrm{C}(3)-\mathrm{N}(2)-\mathrm{Mo}(1) \quad-176.89(15) \\
& \mathrm{C}(1)-\mathrm{N}(1)-\mathrm{N}(2)-\mathrm{C}(3) \quad 0.2(2) \\
& \mathrm{B}(1)-\mathrm{N}(1)-\mathrm{N}(2)-\mathrm{C}(3) \quad 179.44(18) \\
& \mathrm{C}(1)-\mathrm{N}(1)-\mathrm{N}(2)-\mathrm{Mo}(1) \quad 177.20(13) \\
& \mathrm{B}(1)-\mathrm{N}(1)-\mathrm{N}(2)-\mathrm{Mo}(1) \quad-3.6(2) \\
& \text { C(10)-Mo(1)-N(2)-C(3) 31.72(19) } \\
& \mathrm{C}(11)-\mathrm{Mo}(1)-\mathrm{N}(2)-\mathrm{C}(3) \quad-51.04(19) \\
& \mathrm{C}(13)-\mathrm{Mo}(1)-\mathrm{N}(2)-\mathrm{C}(3) \quad 137.6(2) \\
& \mathrm{N}(6)-\mathrm{Mo}(1)-\mathrm{N}(2)-\mathrm{C}(3) \quad-138.72(19) \\
& \mathrm{N}(4)-\mathrm{Mo}(1)-\mathrm{N}(2)-\mathrm{C}(3) \quad 135.07(19) \\
& \text { C(14)-Mo(1)-N(2)-C(3) 65.6(2) } \\
& \mathrm{C}(12)-\mathrm{Mo}(1)-\mathrm{N}(2)-\mathrm{C}(3) \quad-106.9(2) \\
& \text { C(10)-Mo(1)-N(2)-N(1) - } \quad-144.35(15) \\
& \mathrm{C}(11)-\mathrm{Mo}(1)-\mathrm{N}(2)-\mathrm{N}(1) \quad 132.88(15) \\
& \mathrm{C}(13)-\mathrm{Mo}(1)-\mathrm{N}(2)-\mathrm{N}(1) \quad-38.5(3) \\
& \mathrm{N}(6)-\mathrm{Mo}(1)-\mathrm{N}(2)-\mathrm{N}(1) \quad 45.21(14) \\
& \mathrm{N}(4)-\mathrm{Mo}(1)-\mathrm{N}(2)-\mathrm{N}(1) \quad-41.00(14) \\
& \text { C(14)-Mo(1)-N(2)-N(1) -110.43(16) } \\
& \text { C(12)-Mo(1)-N(2)-N(1) 77.1(2) } \\
& \mathrm{C}(5)-\mathrm{C}(4)-\mathrm{N}(3)-\mathrm{N}(4) \quad-0.3(2)
\end{aligned}
$$




\begin{tabular}{|c|c|}
\hline $\mathrm{C}(5)-\mathrm{C}(4)-\mathrm{N}(3)-\mathrm{B}(1)$ & $-174.9(2)$ \\
\hline $\mathrm{N}(5)-\mathrm{B}(1)-\mathrm{N}(3)-\mathrm{C}(4)$ & $-122.0(2)$ \\
\hline $\mathrm{N}(1)-\mathrm{B}(1)-\mathrm{N}(3)-\mathrm{C}(4)$ & 121.1(2) \\
\hline $\mathrm{N}(5)-\mathrm{B}(1)-\mathrm{N}(3)-\mathrm{N}(4)$ & $63.9(2)$ \\
\hline $\mathrm{N}(1)-\mathrm{B}(1)-\mathrm{N}(3)-\mathrm{N}(4)$ & $-53.0(2)$ \\
\hline $\mathrm{C}(5)-\mathrm{C}(6)-\mathrm{N}(4)-\mathrm{N}(3)$ & $0.5(2)$ \\
\hline $\mathrm{C}(5)-\mathrm{C}(6)-\mathrm{N}(4)-\mathrm{Mo}(1)$ & $-174.65(15)$ \\
\hline $\mathrm{C}(4)-\mathrm{N}(3)-\mathrm{N}(4)-\mathrm{C}(6)$ & $-0.1(2)$ \\
\hline $\mathrm{B}(1)-\mathrm{N}(3)-\mathrm{N}(4)-\mathrm{C}(6)$ & $175.00(18)$ \\
\hline $\mathrm{C}(4)-\mathrm{N}(3)-\mathrm{N}(4)-\mathrm{Mo}(1)$ & $175.85(13)$ \\
\hline $\mathrm{B}(1)-\mathrm{N}(3)-\mathrm{N}(4)-\mathrm{Mo}(1)$ & $-9.0(2)$ \\
\hline $\mathrm{C}(10)-\mathrm{Mo}(1)-\mathrm{N}(4)-\mathrm{C}(6)$ & $-58.2(2)$ \\
\hline $\mathrm{C}(11)-\mathrm{Mo}(1)-\mathrm{N}(4)-\mathrm{C}(6)$ & $170.7(5)$ \\
\hline $\mathrm{N}(2)-\mathrm{Mo}(1)-\mathrm{N}(4)-\mathrm{C}(6)$ & $-137.9(2)$ \\
\hline $\mathrm{C}(13)-\mathrm{Mo}(1)-\mathrm{N}(4)-\mathrm{C}(6)$ & $43.0(2)$ \\
\hline $\mathrm{N}(6)-\mathrm{Mo}(1)-\mathrm{N}(4)-\mathrm{C}(6)$ & $142.7(2)$ \\
\hline $\mathrm{C}(14)-\mathrm{Mo}(1)-\mathrm{N}(4)-\mathrm{C}(6)$ & $5.9(2)$ \\
\hline $\mathrm{C}(12)-\mathrm{Mo}(1)-\mathrm{N}(4)-\mathrm{C}(6)$ & $60.3(2)$ \\
\hline $\mathrm{C}(10)-\mathrm{Mo}(1)-\mathrm{N}(4)-\mathrm{N}(3)$ & $127.22(14)$ \\
\hline $\mathrm{C}(11)-\mathrm{Mo}(1)-\mathrm{N}(4)-\mathrm{N}(3)$ & $-3.9(6)$ \\
\hline $\mathrm{N}(2)-\mathrm{Mo}(1)-\mathrm{N}(4)-\mathrm{N}(3)$ & $47.50(14)$ \\
\hline $\mathrm{C}(13)-\mathrm{Mo}(1)-\mathrm{N}(4)-\mathrm{N}(3)$ & $-131.63(15)$ \\
\hline $\mathrm{N}(6)-\mathrm{Mo}(1)-\mathrm{N}(4)-\mathrm{N}(3)$ & $-31.88(14)$ \\
\hline $\mathrm{C}(14)-\mathrm{Mo}(1)-\mathrm{N}(4)-\mathrm{N}(3)$ & $-168.65(14)$ \\
\hline $\mathrm{C}(12)-\mathrm{Mo}(1)-\mathrm{N}(4)-\mathrm{N}(3)$ & $-114.26(14)$ \\
\hline $\mathrm{C}(8)-\mathrm{C}(7)-\mathrm{N}(5)-\mathrm{N}(6)$ & $0.6(2)$ \\
\hline $\mathrm{C}(8)-\mathrm{C}(7)-\mathrm{N}(5)-\mathrm{B}(1)$ & $166.6(2)$ \\
\hline
\end{tabular}




$$
\begin{aligned}
& \mathrm{N}(1)-\mathrm{B}(1)-\mathrm{N}(5)-\mathrm{C}(7) \quad-108.5(2) \\
& \mathrm{N}(3)-\mathrm{B}(1)-\mathrm{N}(5)-\mathrm{C}(7) \\
& \mathrm{N}(1)-\mathrm{B}(1)-\mathrm{N}(5)-\mathrm{N}(6) \quad 56.2(2) \\
& \mathrm{N}(3)-\mathrm{B}(1)-\mathrm{N}(5)-\mathrm{N}(6) \quad-60.9(2) \\
& \mathrm{C}(8)-\mathrm{C}(9)-\mathrm{N}(6)-\mathrm{N}(5) \quad-0.8(2) \\
& \mathrm{C}(8)-\mathrm{C}(9)-\mathrm{N}(6)-\mathrm{Mo}(1) \quad-171.25(15) \\
& \mathrm{C}(7)-\mathrm{N}(5)-\mathrm{N}(6)-\mathrm{C}(9) \quad 0.1(2) \\
& \mathrm{B}(1)-\mathrm{N}(5)-\mathrm{N}(6)-\mathrm{C}(9) \quad-167.35(18) \\
& \text { C(7)-N(5)-N(6)-Mo(1) 171.64(13) } \\
& \mathrm{B}(1)-\mathrm{N}(5)-\mathrm{N}(6)-\mathrm{Mo}(1) \\
& \text { C(10)-Mo(1)-N(6)-C(9) 96.6(3) } \\
& \mathrm{C}(11)-\mathrm{Mo}(1)-\mathrm{N}(6)-\mathrm{C}(9) \quad 27.53(19) \\
& \mathrm{N}(2)-\mathrm{Mo}(1)-\mathrm{N}(6)-\mathrm{C}(9) \quad 123.36(19) \\
& \text { C(13)-Mo(1)-N(6)-C(9) -76.30(19) } \\
& \mathrm{N}(4)-\mathrm{Mo}(1)-\mathrm{N}(6)-\mathrm{C}(9) \quad-156.12(19) \\
& \mathrm{C}(14)-\mathrm{Mo}(1)-\mathrm{N}(6)-\mathrm{C}(9) \quad-78.4(2) \\
& \text { C(12)-Mo(1)-N(6)-C(9) -46.40(18) } \\
& \mathrm{C}(10)-\mathrm{Mo}(1)-\mathrm{N}(6)-\mathrm{N}(5) \quad-72.6(3) \\
& \text { C(11)-Mo(1)-N(6)-N(5) -141.67(15) } \\
& \mathrm{N}(2)-\mathrm{Mo}(1)-\mathrm{N}(6)-\mathrm{N}(5) \quad-45.84(14) \\
& \text { C(13)-Mo(1)-N(6)-N(5) 114.49(15) } \\
& \mathrm{N}(4)-\mathrm{Mo}(1)-\mathrm{N}(6)-\mathrm{N}(5) \quad 34.68(15) \\
& \text { C(14)-Mo(1)-N(6)-N(5) 112.39(15) } \\
& \text { C(12)-Mo(1)-N(6)-N(5) 144.39(15) } \\
& \mathrm{C}(13)-\mathrm{C}(12)-\mathrm{O}(3)-\mathrm{C}(21) \quad 0.1(3) \\
& \mathrm{C}(16)-\mathrm{C}(12)-\mathrm{O}(3)-\mathrm{C}(21) \quad 152.46(18) \\
& \mathrm{Mo}(1)-\mathrm{C}(12)-\mathrm{O}(3)-\mathrm{C}(21) \quad-73.1(2)
\end{aligned}
$$



$\mathrm{C}(14)-\mathrm{C}(15)-\mathrm{O}(5)-\mathrm{C}(16)$
$-50.8(2)$
$\mathrm{C}(17)-\mathrm{C}(15)-\mathrm{O}(5)-\mathrm{C}(16)$
$75.1(2)$
$\mathrm{C}(12)-\mathrm{C}(16)-\mathrm{O}(5)-\mathrm{C}(15)$
$44.8(2)$
$\mathrm{C}(20)-\mathrm{C}(16)-\mathrm{O}(5)-\mathrm{C}(15)$
$-78.8(2)$

\section{REFERENCES}

1. Sloan, T. E., Top. Stereochem. 1981, 12, 1-36.

2. Yin, J.; Liebeskind, L. S., J. Am. Chem. Soc. 1999, 121, 5811-5812.

3. Moretto, A. F.; Liebeskind, L. S., J. Org. Chem. 2000, 65, (22), 7445-7455.

4. Malinakova, H. C.; Liebeskind, L. S., Org. Lett. 2000, 2, (24), 3909-3911.

5. The full details of the Mukaiyama-Michael additions to $\eta 3$-pyranyl and $\eta 3$-pyridinylmolybdenum $\pi$ complexes will be published in a full paper together with Mukaiyama aldol reactions.

6. SMART Version 5.628, 2003, Bruker AXS, Inc., Analytical X-ray Systems, 5465 East Cheryl Parkway, Madison WI 53711-5373.

7. SAINT Version 6.36A, 2002, Bruker AXS, Inc., Analytical X-ray Systems, 5465 East Cheryl Parkway, Madison WI 53711-5373.

8. SADABS Version 2.08, 2003, George Sheldrick, University of Göttingen.

9. SHELXTL V6.12, 2002, Bruker AXS, Inc., Analytical X-ray Systems, 5465 East Cheryl Parkway, Madison WI 53711-5373.

10. A. J. C. Wilson (ed), International Tables for X-ray Crystallography, Volume C. Kynoch, Academic Publishers, Dordrecht, 1992, Tables 6.1.1.4 (pp. 500-502) and 4.2.6.8 (pp. 219-222). 